\title{
54th ANNUAL SASKATCHEWAN CHRISTMAS BIRD COUNT — 1995
}

\section{Compiled by Wayne C. Harris, Saskatchewan Environment and Resource Management, 350 Cheadle Street West, Swift Current, SK. S9H 4G3}

More traditional winter weather returned for the 1995 count. Snow cover was general throughout and temperatures were moderate during the count period (16 December 1995 through 2 January 1996). The 86 counts completed this year were two less than last year, continuing a gradual decline since the record 99 in 1991. In spite of the return to more traditional winter weather from the balmy days of 1994, the number of people participating increased from 597 in 1994 to 729 this year. The number of counts was down, but the increase in the number of people participating brought the number of hours accumulated up from 1,120 last year to 1,272 .

\section{Weather and Coverage}

Table 1 summarizes the weather conditions reported on the counts. As indicated, the temperatures were colder than last year, but still above the normal, with the average overnight low being $-15^{\circ} \mathrm{C}$ and the daytime highs averaging $-6^{\circ} \mathrm{C}$. Kamsack had the coldest night at $-41^{\circ} \mathrm{C}$ while Chitek Lake reached a high of $+3^{\circ} \mathrm{C}$. The heaviest snow cover was again on the east side of the province. The average snow depth for the entire province was almost $30 \mathrm{~cm}$.

\section{The Birds}

Ninety-three species were recorded on count day, with an additional three species during the count period, giving an overall total of 96 species, down two from last year. The results are compiled in Tables 3 , 4 and 7 . The total number of birds seen totalled 97,585 individuals, down $13 \%$ from last year's 112,685 . The most abundant species was House Sparrow with 16,919 individuals, followed by the Snow Bunting with 16,021 . Surprisingly common, in spite of general snow cover, was the Mallard $(12,217)$. No other species exceeded 10,000 individuals.

Population trends of some common species are provided in Table 5. The most substantial increases were for irruptive forest species. Among these, Red-breasted Nuthatches were the most prominent but redpolls, grosbeaks and crossbills were also in above-average numbers. Increases in grouse numbers were also evident, especially Gray Partridge and Sharp-tailed Grouse.

Raptor populations declined. The only exception to this was Northern Goshawks that were up, following increasing hare and grouse numbers. Although the decline in Bald Eagles is not surprising with the return of colder temperatures, that of Golden Eagles is. Following the Golden Eagle's downward pattern are Merlin, Prairie Falcon, Rough-legged Hawk, Short-eared Owl, Snowy Owl and even Great Horned Owl.

Noticeable declines were also found in waxwing, raven and magpie 
populations. Though they were the most common species, House Sparrows continue their long-term decline.

\section{New Species}

Three new species and one new subspecies were found this year, bringing the all-time list to 162 species. The first was a Lewis' Woodpecker found at Fort Qu'Appelle. Seen by several people on count day, it had not been seen before and was not found again afterwards. Rare at any time of year, this is an exceptional find.

The Yellow-headed Blackbird was seen at Moose Jaw 22 December but did not remain until count day. This is surprisingly the first time this species has been found on a count and completed the list of blackbirds (normally found during summer) for the winter list.

The last new species was a Rosebreasted Grosbeak reported from Love-Torch River. Although common during the summer, this species normally winters in the tropics.

The new subspecies was a Harlan's Red-tailed Hawk at White Bear. This bird has been present at the Jordheim farm since 24 November apparently living on the large flock of Rock Doves present.

\section{Rare Species}

Table 4 lists the least frequent species on the counts and the rarest species are all in this table. The count period Ring-necked Duck at Fort Qu'Appelle was only the second count record. A Lincoln's Sparrow at Broadview was the first time the species was seen on count day, with the two previous records both being count period records. Other rarities included an Eastern Screech-owl at Estevan that responded to a tape of screech-owl calls, providing only the second count record. Varied Thrushes found at both Saskatoon and Emma Lake equalled last year's two records. The Rufous-sided Towhee found at Skull Creek for the second year in a row has become either a permanent resident or this locale provides the optimum in Saskatchewan winter habitat for the species. Other unusual species included a White-winged Scoter at Pike Lake, Long-eared Owl at Skull Creek, Song Sparrow at Turtle Lake and American Goldfinch at both Shaunavon and Craven.

House Finches were reported from seven separate counts and the previous high count of 22 was doubled by Moose Jaw. The cold extremes of our winters are obviously not going to stop the spread of this species that has expanded rapidly westward from eastern North America in the past ten years.

\section{High Counts}

Table 6 provides a summary of new high counts recorded for species during the 1995 count. Gardiner Dam continues its prominence in merganser counts with a new high of 438 Common Mergansers. Saskatoon is earning the title of feeder capital of Saskatchewan, with an impressive list of new high counts that are probably a result of feeders. The new highs from Saskatoon include Northern Flicker, Blue Jay, Blackbilled Magpie, Black-capped Chickadee, and Red-breasted Nuthatch. They also broke the 20-year-old record for White-winged Crossbill, part of a massive invasion of this species across the province. Other impressive counts include 23 Whitebreasted Nuthatches at Snowden, 
250 Dark-eyed Juncos at Grasslands National Park and 214 Pine Siskins at Prince Albert.

\section{Count Areas and Participants}

Names of compilers are in italics. Number of participants in each count are in parentheses following the names.

1. ARMIT. Anne Harris, Valeri Harris, Wayne Harris, Sheila Lamont. (4)

2. ASSINIBOIA. Ed Bearss, Jack Burgeson, Cecil Hayward, Nelson Lamb, Delmar Pettem, Wilf Prentice. (6)

3. BANGOR. Jean Hilton. (1)

4. BETHUNE. Doug and Vera Laing. (2)

5. BIGGAR. Kyle Peiffer, Guy Wapple, Robert Wapple, Liana Warke. (4)

6. BIRCH HILLS. Marg Mareschal, Moe Mareschal, Don Weidl. (3)

7. BRIGHTWATER RESERVOIR. Andy Didiuk, Alan Smith. (2)

8. BROADVIEW. Dave Chaskavich. (1)

9. CABRI. Carman Dodge, Henri Lebastard. (2)

10. CANDLE LAKE. Burke Korol, John Korol, Helen Korol. (3)

11. CHITEK LAKE. Marcel Cornect. (1)

12. CHRISTOPHER LAKE. Dorothy Bird. (1)

13. CLARK'S CROSSING. Nancy Allan, Evelyn Bacon, Barbara Brokx, Emile Brokx, Muriel Carlson, Fran Eldridge, Mary Gilliland, Bernie Gollop, Bob Johanson, Marlene Kalanack, Kay Krueger, Gerard Lahey, Arnold MacMillan, Cliff Matthews, Brian McGill, Vance McNab, Mack Miller, Menno Nickel, Hilda Noton, Keith Pahl, Betty Penner, Stan Shadick, Mary Shoard, Jim Wedgwood, Michael Williams, Jim Wood, Lois Wooding. (27)

14. CORONACH. Wayne Harris, Tim Moxham. (2)

15. CRAVEN. Margaret Belcher, Al Binney, Betty Binnie, Trevor Herriot (non-participating compiler), Phil Holloway, Bob Kreba, Ron Myers, Bill Ogilvie, Pat Ogilvie, Curtis Pollock. (9)

16. CROOKED LAKE. Warren Hjertaas, Bill Livesay, Mayta Livesay, Boyd Metzler, Dorothy Skene, Ed Skene, Mel Zimmer. (7)

17. CROOKED RIVER. Karl Mehler, Katherine Mehler, Kristine Mehler, Margaret Mehler, Morley Mehler, Phyllis Siemens. (6)

18. DILKE. Margaret Belcher, Brian McArton, Ken McArton. (3)

19. DUVAL. Enid Cumming, George Herber, Linda Korytko, lain Richardson, Lloyd Saul, Mez Smith. (6)

20. EASTEND. Dorothy Holmes, Henri Lebastard, Florence McCuaig, Laureen McLeod, Helen Wlaz. (5)

21. EMMA LAKE. Glen Hanson, Jean Hanson, Deanna Krug, Norman Krug. (4)

22. ENDEAVOUR. Norman Harris. (1)

23. ESTEVAN. Gord Tenold, Henrietta Tenold, Guy Wapple. (3)

24. FENTON. Carman Dodge. (1)

25. FIFE LAKE. Martin Myers, Robert Rafuse. (2)

26. FORT QU'APPELLE. Elizabeth Aitken, James Armstrong, Peter Ashcroft, Phyllis Bordass, William Bordass, Errol Cochrane, Vera Cousins, Anne Davies, Frank Davies, Doug Harman, Ronald Hooper, Peter Horsman, Lois Lamontagne, George Larocque, Lucy Larocque, Lloyd Leader, Maurice Lindgren, Jack Lowe, Don McDougall, Jean McKenna, Alan Mlazgar, Webb Palmer, Paul Paquin, Helen Reimer, Lorne Rowell, Lloyd Talbot, Gus Vanderpolder. (27)

27. FORT WALSH. Anne Harris, Valeri Harris, Wayne Harris, Burke Korol, Sheila Lamont, Sue McAdam, Wilkes Parsonage, Gary Provanchier, Guy Wapple, Robert Wapple. (9)

28. GARDINER DAM. Anne Harris, Valeri Harris, Wayne Harris, Mary Houston, Stuart Houston, Ron Jensen, Sheila Lamont, Stuart Slattery, Jantina Portman, Guy Wapple, Robert Wapple, Dan Zazelenchuk. (12) 
29. GOOD SPIRIT LAKE. Bill Anaka, Joyce Anaka, Julia Wiwchar. (3)

30. GOVENLOCK. Anne Harris, Valeri Harris, Wayne Harris, Sheila Lamont, Sue McAdam, Guy Wapple, Robert Wapple. (7)

31. GRASSLANDS NATIONAL PARK. Wayne Harris, Debbie Kilfoyle. (2)

32. GRASSLANDS NATIONAL PARK (NW). Brian Besler, Pat Fargey, Sherri Hohn, Florence Miller. (4)

33. HEPBURN. Phyllis Siemens. (1)

34. HORSESHOE BEND (North of Kinistino). Davene Berg, Dannelle Messer, Stacii Messer, Verna Messer. (4)

35. HUMBOLDT. Ed Brockmeyer, Mike Volk. (2)

36. INDIAN HEAD. Irv Escott, David Gehl, Gordon Howe, Dora Nichols, Norine Nichols, Lorne Scott. (6)

37. KAMSACK. John Berisoff, George and Mary Bernard, Agnes Betz, Marlen Brock, Mable Buceuk, Lindee Dewores, Barb Elasser, Jay and Lena Fry, Anita Klochko, Bill Koroluk, Laura Loeppky, W.J.C. May, Adeline Nykolaishen, Helen Panchuk, Dallas, Kelsey and Ryley Rezansoff, Isabel Ritchie, Metro Rosowsky, Eve Sasyniuk, David and Elsie Severson, John Solmon, Elenor Sookochetz, Stan Stone, Joyce and Pete Uhlow, Gordon Woloschuk. (30)

38. KELVINGTON. Pat Finnie, Dianne Sloan, Marguerite Sloan. (3)

39. KENASTON. Doug Beckie, Lawrence Beckie. (2)

40. KILWINNING. Alan Daku, Ed Driver, Marg Driver, Doug Martin. (4)

41. KINDERSLEY. Gerry Essar, Michael Essar. (2)

42. KINLOCH. Don Forbes, Doreen Forbes, Cliff Logan, Wilf Rodenberg. (4)

43. KUTAWAGAN LAKE. Wayne Harris, Alan Rahn. (2)

44. LARONGE. Jim Paul, Lorie Ann Paul. (2)

45. LAST MOUNTAIN LAKE N.W.A. John Dunlop, Anne Harris, Valeri Harris,
Wayne Harris, Sheila Lamont, Lois Vanthuyne. (6)

46. LEADER (North). Daisy Meyers. (1)

47. LEADER (South). John Flood, John Rauch, Brenda Schnell, Susan Springett. (4)

48. LIVELONG. Sarah Pavka. (1)

49. LOVE-TORCH RIVER. Hal Birkett, Joan Birkett, Laurann Carr, Bert Dalziel, Duke Dalziel, Joan Dalziel, Kari Dalziel, Nora Dalziel, Sara Dalziel, Anita Deutschmann, Eric Deutschmann, Rita Deutschmann, Ed Dixon, Marlene Dixon, Betty Donovan, Bruce Donovan, Sean Donovan, Troy Donovan, Eileen L'Heureux, Mildred Long, Bill Matthews, Lynn Matthews. (22)

50. LUSELAND. Kim Finley, Estelle Finley, Graeme Finley, Liam Finley, Bill Frey, Brent Honeker, Dustin Honeker, Herman Honeker, Levi Honeker, Shirley Honeker. (10)

\section{MACDOWALL. Myron Barton. (1)}

52. MEADOW LAKE. Bill Caldwell, Janet Caldwell, Byron Golly, Stuart Golly, Tyler Golly, Anne Sequin, Randy Sequin, Robbie Sequin, John Weir, Andrew Wilson, Bob Wilson, Cindy Wilson, Ian Wilson. (13)

53. MELFORT. Dave Atamanchuk, Phil Curry, Kim Eskowich, Kate Johnson, Gerri Knudson, Gerard Letain, Dave Pochailo. (7)

54. MELFORT (SE). Frieda Markland. (1)

55. MELVILLE. Bill Barmry, Marion MacLean, Jeanette Olson, Ross Wotherspoon. (4)

56. MISSINIPE. Jim Bot, Karen Coleman, Shirley Glass. (3)

57. MOOSE JAW. Ron and Vi Anderson, Edith Bell, Nora Bowler, Doug and Helen Brunsdon, Brian Butlin, Elsie Carrick, Barry and Fern Dowse, Ed, Hartley and Ken Fredeen, Bill and Evelyn Gilmour, George and Margaret Grigg, Al, David, Terry and Trevor Gurnsey, Kerry and Robert Hanley, Bob, Pam and Pat Kern, Eve King, Cy and Leith Knight, T. Lowe, Connie and Hugh Mclntyre, Enid and Ernie Meadows, Mary Montague, Tom Mulligan, Wilma Pickering, Ramsay and Marj Ross, Gavina Reekie, Irene Reid, Gus and Mike Sagal, Glen 
Steel, Alice and Glen Urquhart, Arie Van Dorland, Ed and Grace Walker. (49)

58. MOOSE MOUNTAIN. Ray Belanger, Greg Bobbitt, Dick Gutfriend, Boyd Metzler, John Pollock, Keith Sakatch. (6)

59. NIPAWIN. Inge Brown, Vi Budd, Joyce Christiansen, Sandra Hosaluk, Doug Pegg, Doug Phillips, Shirley Phillips. (7)

60. PADDOCKWOOD - CHRISTOPHER LAKE. Blake Jones, Cliff Matthews. (2)

61. PIKE LAKE. Nancy Allan, Lawrence Beckie, Mark Bidwell, Carol Blenkin, Kent and Laurel Brace, Doug and Helen Campbell, Ron and Sheila Canning, Muriel Carlson, Betty Ann and Tom Dunlop, Emile and Hanna Erickson, Garry and Kathy Genereux, Bob Girvan, Bernie Gollop, Mike Gollop, Kurt Greenwood, Jim Hay, Judy Hommen, Don Kinzie, Kay Kruger, Joyce Manton, Cliff Matthews, Menno Nickel, Keith Pahl, Joyce Petrie, Gladys and Glen Pippin, Frank Roy, Monte and Pauline Sawyer, Alice Silversides, Ivor Thokel, Hilda Voth, Irma and Tom Watson, Michael Williams, Lois Wooding, (42)

62. PRINCE ALBERT (B). John Burt, Pam Burt, Jamie Chartrand, Carman Dodge, Keith Dodge, Bebe Gilmour, Iris Goebel, Joe Graumans, Jessie Irish, Carmen Lizee, Ethen Lizee, Jasmine Lizee, Jeremie Lizee, Laurent Lizee, Tim Loran, Collin Magee, Jeremy Peters, Ted Snow, Deirdre Todd, Jim Town. (20)

63. PRINCE ALBERT NATIONAL PARK. Ed Brown, Hazel Brown, Susan Carr, Kim Clark, Suzanne Clark, Emily and Greg Fenton, Michael Fitzsimmons, Dan Frandsen, David Henry, Elizabeth Henry, Suzanne Henry, Howard Lancaster, Karen Larsen, Bradley Muir, Laura Muir, Adam Pidwerbeski, Karen Smith, Elia Tarasoff, Harry Tarasoff, Gavril Tarasoff, Marg Tarleton, Colleen Watson, Libby Weir, Graeme Wesson, Daniel Wesson, Kristin Wesson. (27)

64. QU'APPELLE VALLEY DAM. Jordan Hamm, Roy John, Kay Krueger, Mack Miller, Bob Plaster, Frank Roy, Stan Shadick, Michael Williams, Jim Wood. (9)
65. RAYMORE. Anne Harris, Valeri Harris, Wayne Harris, Sheila Lamont. (4)

66. REGINA. Sandy Ayer, Jessie Bailey, Donna Barclay, Margaret Belcher, Lionel Bonneville, Stephane Bonneville, David Bryant, Wanda Calvin, Andy Delyk, Scott Dickin, Jim Elliot, Robert Ewart, Sandra Ewart, Kay Ferguson, Mary Ferguson, Margaret Filden, Jill Forrester, Alana Francis, Steven Francis, Dale Hjertaas, Paule Hjertaas, Bob Kreba, Linda Langenbacher, Clare Lepage, Don Lepage, Bob Luterbach, Lauren Mang, Linda McDowell, Ron Meyers, Dieter Peschken, John Pollock, Brian Rainey, Pat Sargeant, Margaret Skeel, Frank Switzer, Dan Vetter, Cherie Westmoreland. (37)

67. ROCKGLEN-BORDERLAND. Martin Meyers, Bob Rafuse. (2)

68. ROUND LAKE (Qu'Appelle Valley). Pat Connoly, Doug Francis, Boyd Metzler, John Pollock. (4)

69. ROUND LAKE (Prince Albert). Evelyn Marshall. (1)

70. SASKATCHEWAN LANDING PROVINCIAL PARK. Wayne Harris, Ron Jensen, Jeffrey Jensen, Wayne Luchinski. (4)

71. SASKATOON. Don Adams, Darlene Aikman, Nancy Allan, Tony Allen, Juhachi Asai, Kengo Asai, Sumiko Asai, Don Beazely, Beverly Beland, Orval Beland, Bob Besant, Joyce Besant, Mark Bidwell, Eveline Boudreau, Gail Bunt, Muriel Carlson, Bill Cates, Louise Cook, Adam Cooper, Leah Currie, Odile Daku, Eileen Dalglish, Ed Driver, Anne Dzus, Fran Eldridge, Melanie Elliott, Helen Fast, Martin Gerard, Silvia Gerard, Mary Gilliland, Bernie Gollop, Madeleine Gollop, Mike Gollop, Jerry Haigh, John Hanbidge, Kaija Harris, Mary Houston, Stuart Houston, Roy John, Marlene Kalanack, Richard Kerbes, Gordon Koshinsky, Margaret Koshinsky, Barbara Kozmyk, Gerard Lahey, Felicity Lane, Louise Roy Mark, John Mason, Josephine Mason, Cathryn Miller, David Miller, Garth Nelson, Menno Nickel, Bruce Noton, Hilda Noton, Phil Norton, Keith Pahl, Ann Pocknell, Wilf Ready, Lynn Rowland, Orion Schille, Jim Smart, Al Smith, Joe Sondershausen, Inge Tabel, Phil Taylor, Barry Theissen, Ivor Thokle, Ron Trischuk, Hilda Voth, Heather Wagg, Jim Wedgwood, George West, Michael 
Williams, Jim Wood, Dan Zazelenchuk. (77)

72. SCOTT. Alec Elmquist, Guy Wapple, Robert Wapple. (3)

73. SHAMROCK. Hugh Henry. (1)

74. SHAUNAVON. Carl Osterberg, Marlene Osterberg, Ruth Robertson, Fred Wall. (4)

75. SKULL CREEK. Eileen Bennetto, Jim Bennetto, Ray Bennetto, Doris Bircham, Ralph Drever, Bob Eccleston, Betty Mann, Joane Peterson, Cory Wasilow, Harvey Wasilow, Mark Wasilow, Robin Wolfater. (12)

76. SNOWDEN. Bonnie Bailey, Allan and Rita Birhenthal, Bert Dalziel, Diane and Don Frieson, Irene Hagel, Karen Hagel, Ralph and Jenny Johnson, Violet Lien, Lorne and Sharon Lindberg, Betty and Harold Pagan, Edna and Percy Pagan, Jack Pickett, Bill and Vera Schemenauer, Beu Smearr, Harold and Irene Thompson, Irene White. (24)

77. SPALDING. Bill Spizawka, Velma Spizawka. (2)

78. SPINNEY HILL. Ed Driver, Tony Goulding. (2)

79. SQUAW RAPIDS. Anne Harris, Valeri Harris, Wayne Harris, Sheila Lamont, Guy Wapple. (5)

80. SWIFT CURRENT. James Beattie, Michelle Gendreau, Hugh Henry, Julie Jensen, Ron Jensen, Walt Kreuger, Arlyne Lawson, Doug Lawson, Laure
Neish, Ted Philipchuck, Mike Rogers, Scott Rogers, Richard Schaitel, Don Sluth, Floyd Stinson, Irene Stinson, Doris Thoreson, Kaye Waters, John Weston, Pearl Weston. (20)

81. TISDALE. Carl Mohr, Joyce Mohr. (2)

82. TURTLE LAKE. Steve and Carol Burand, Muriel Carlson, Adam Cooper, Gil Heim. (5)

83. WEYBURN. Leo Belanger, Louise Belanger, Ray Belanger, Sophie Belanger, Greg Bobbitt, Jace Brown, Ross Douglas, Norm Flaten, Harry Kapusianyk, Norma Kapusianyk, Tanya Laurent, Betty Layh, Phil Layh, Rhode Moldenhauer, Elizabeth Murray, John Murray, Nick Postey, Keith Sakatch, Stew Stairmand, Joe Weisgerber. (20)

84. WHITE BEAR. Daryl Jordheim, Floyd Jordheim, Gary Jordheim, Laine Jordheim, Sig Jordheim, Greg MacCulloch, Lynette MacCulloch. (7)

85. WHITEWOOD. Cliff Ashfield, Kurtis Briggs, Pat Finkas, Vangie Hoggarth, Dallyn Holmstrom, Joyce Jordan, Wilfred Jordens, Bernice Juzyniec, Felix Juzyniec, Mavis Kay, Jake Kendall, Joyce Kidd, Doreen McPhail, Jean Meadows, Bill Meszaros, Illa Meszaros, Boyd Metzler, Marlene Rey, Tony Rey, Len Santo, Howard Shurvin, Vera Shurvin, Diane Veresh, Justin Westberg. (24)

86. YORKTON. Warren and Elinor Hjertaas. (2)

As the human wave rolled over the last of the virgin islands like a smothering blanket, Paleo-Indians throughout America, Polynesians across the Pacific, Indonesians into Madagascar, Dutch sailors ashore on Mauritius (to meet and extirpate the dodo), they were constrained by neither knowledge of endemicity nor any ethic of conservation. For them the world must have seemed to stretch forever beyond the horizon. If fruit pigeons and giant tortoises disappear from this island, they will surely be found on the next one. What counts is food today, a healthy family, and tribute for the chief, victory celebrations, rites of passage, feasts. As the Mexican truck driver said who shot one of the last two imperial woodpeckers, largest of the world's woodpeckers, "It was a great piece of meat." E.O. Wilson, 1992. The diversity of life. W.W. Norton and Company, New York. 424 pp. 


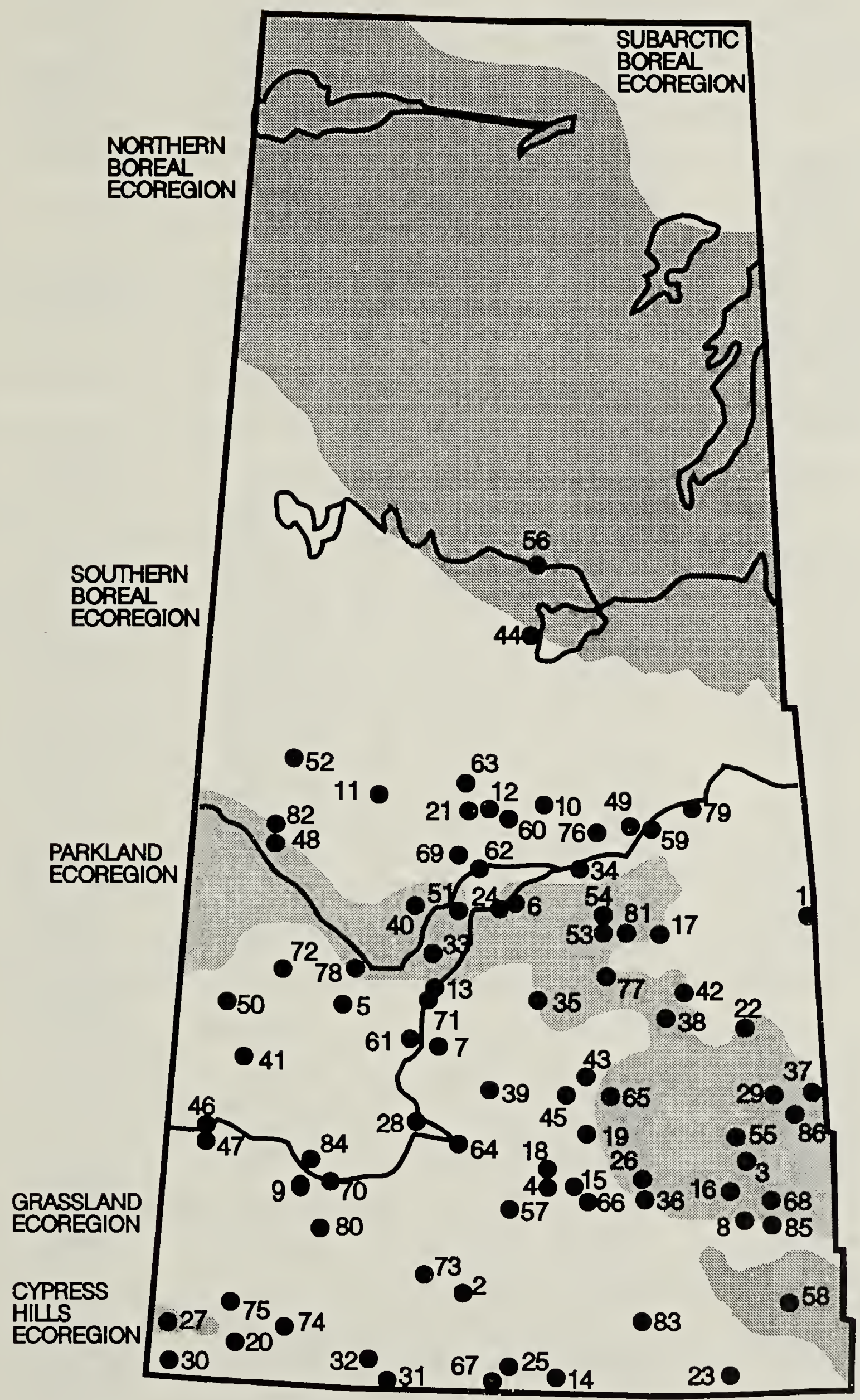

Figure 1. Location of 1995 counts (numbers correspond to locality names in Tables). 


\section{Table 1: COUNT WEATHER CONDITIONS (Temperature ${ }^{\circ} \mathrm{C}$, Wind in $\mathrm{kmph}$}

and Snow Cover in $\mathrm{cm}$.)

\begin{tabular}{|c|c|c|c|c|c|c|c|c|}
\hline Locality & \begin{tabular}{|c|} 
Min. \\
Temp \\
\end{tabular} & $\begin{array}{c}\text { Max. } \\
\text { Temp } \\
\end{array}$ & $\begin{array}{l}\text { Min. } \\
\text { Wind } \\
\end{array}$ & $\begin{array}{l}\text { Max. } \\
\text { Wind } \\
\end{array}$ & $\begin{array}{l}\text { Min. } \\
\text { Snow }\end{array}$ & $\begin{array}{l}\text { Max. } \\
\text { Snow } \\
\end{array}$ & AM Sky & PM Sky \\
\hline 1. ARMIT & -22 & -11 & 10 & 40 & 30 & 40 & overcast & overcast \\
\hline 2. ASSINIBOIA & -25 & -20 & 2 & 5 & 30 & 40 & clear & clear \\
\hline 3. BANGOR & -10 & -8 & 15 & 18 & 40 & 45 & mostly clear & clear \\
\hline 4. BETHUNE & -14 & -10 & 0 & 15 & 12 & 14 & overcast & mostly cloudy \\
\hline 5. BIGGAR & -11 & -7 & 8 & 20 & 0 & 30 & partly cloudy & partly cloudy \\
\hline 6. BIRCH HILLS & -6 & -5 & 5 & 5 & 20 & 35 & $\begin{array}{l}\text { overcast; } \\
\text { light snow }\end{array}$ & $\begin{array}{l}\text { overcast; } \\
\text { light snow } \\
\end{array}$ \\
\hline $\begin{array}{l}\text { 7. BRIGHTWATER } \\
\text { RESERVOIR } \\
\end{array}$ & -15 & -10 & 0 & 5 & 5 & 15 & overcast & clear \\
\hline 8. BROADVIEW & -16 & -10 & 0 & 5 & 10 & 50 & clear & clear \\
\hline 9. CABRI & -5 & -2 & & & 5 & 15 & & \\
\hline 10. CANDLE LAKE & -18 & -12 & 0 & 10 & 30 & 50 & overcast & overcast \\
\hline 11. CHITEK LAKE & 0 & 3 & 0 & 5 & & & overcast & overcast \\
\hline $\begin{array}{l}\text { 12. CHRISTOPHER } \\
\text { LAKE (N) }\end{array}$ & -15 & -7 & 0 & 5 & 38 & 70 & mostly clear & mostly clear \\
\hline $\begin{array}{l}\text { 13. CLARK'S } \\
\text { CROSSING }\end{array}$ & -19 & -16 & 0 & 10 & 4 & 60 & partly cloudy & overcast \\
\hline 14. CORONACH & -12 & -3 & 0 & 15 & 0 & 30 & clear & $\begin{array}{l}\text { overcast; } \\
\text { heavy fog }\end{array}$ \\
\hline 15. CRAVEN & -18 & -16 & 0 & 0 & & & $\begin{array}{l}\text { overcast; } \\
\text { light snow }\end{array}$ & \\
\hline 16. CROOKED LAKE & -12 & -10 & 10 & 15 & 40 & 50 & overcast & mostly clear \\
\hline 17. CROOKED RIVER & -7 & -4 & 0 & 0 & 30 & 40 & overcast & overcast \\
\hline 18. DILKE & -6 & -5 & 5 & 15 & 0 & 60 & overcast & overcast; light fog \\
\hline 19. DUVAL & -8 & -4 & $\underline{0}$ & 9 & 2 & 35 & clear & partly cloudy \\
\hline 20. EASTEND & & & & & & & mostly clear & partly cloudy \\
\hline 21. EMMA LAKE & -12 & -8 & 0 & 5 & 40 & 60 & overcast & partly cloudy \\
\hline 22. ENDEAVOUR & -9 & -4 & 0 & 5 & 20 & 30 & partly cloudy & mostly clear \\
\hline 23. ESTEVAN & -14 & -8 & 0 & 10 & 0 & 15 & $\begin{array}{l}\text { overcast; } \\
\text { moderate fog }\end{array}$ & partly cloudy \\
\hline 24. FENTON & -14 & -2 & 0 & 5 & 10 & 30 & clear & \\
\hline 25. FIFE LAKE & -15 & -10 & 15 & 20 & 20 & 25 & partly cloudy & partly cloudy \\
\hline 26. FORT QU'APPELLE & -12 & -10 & 0 & 0 & 12 & - & overcast & partly cloudy \\
\hline 27. FORT WALSH & -15 & -5 & 0 & 10 & 5 & 40 & mostly clear & mostly clear \\
\hline 28. GARDINER DAM & -21 & -11 & 20 & 50 & 10 & 40 & partly cloudy & overcast \\
\hline 29. GOOD SPIRIT LAKE & -13 & -7 & 15 & 20 & 18 & 27 & overcast & overcast; light fog \\
\hline 30. GOVENLOCK & -20 & -8 & 0 & 20 & 5 & 15 & heavy fog & $\begin{array}{l}\text { partly cloudy; } \\
\text { light fog }\end{array}$ \\
\hline $\begin{array}{l}\text { 31. GRASSLANDS } \\
\text { N. PARK }\end{array}$ & -24 & -9 & 0 & 5 & 0 & 10 & clear & clear \\
\hline $\begin{array}{l}\text { 32. GRASSLANDS } \\
\text { N. PARK (NW) }\end{array}$ & -10 & -6 & 0 & 5 & 5 & 15 & $\begin{array}{l}\text { overcast; } \\
\text { moderate fog }\end{array}$ & \\
\hline 33. HEPBURN & -20 & -15 & 0 & 0 & 10 & 30 & clear & clear \\
\hline $\begin{array}{l}\text { 34. HORSESHOE BEND } \\
\text { (N. of Kinistino) }\end{array}$ & -10 & -8 & 0 & 0 & 30 & 40 & overcast; light fog & $\begin{array}{l}\text { overcast; } \\
\text { light snow }\end{array}$ \\
\hline 35. HUMBOLDT & -12 & -8 & 0 & 2 & 10 & 20 & clear & overcast \\
\hline \multicolumn{9}{|l|}{ 36. INDIAN HEAD } \\
\hline 37. KAMSACK & -41 & & 0 & 5 & 120 & & clear & mostly clear \\
\hline 38. KELVINGTON & -18 & -15 & 0 & 5 & 30 & 60 & partly cloudy & mostly clear \\
\hline 39. KENASTON & -16 & -6 & 14 & 16 & 15 & 20 & partly cloudy & mostly clear \\
\hline 40. KILWINNING & -6 & -6 & 0 & 5 & 15 & 40 & clear & mostly clear \\
\hline 41. KINDERSLEY & -10 & -7 & & & 10 & 30 & $\begin{array}{l}\text { partly cloudy; } \\
\text { heavy fog }\end{array}$ & $\begin{array}{l}\text { overcast; } \\
\text { light snow }\end{array}$ \\
\hline 42. KINLOCH & -3 & 2 & 0 & 5 & 32 & 36 & overcast & partly cloudy \\
\hline 43. KUTAWAGAN LAKE & -12 & -10 & 0 & 5 & 0 & 20 & overcast & partly cloudy \\
\hline
\end{tabular}


Table 1: COUNT WEATHER CONDITIONS (continued)

\begin{tabular}{|c|c|c|c|c|c|c|c|c|}
\hline Locality & $\begin{array}{l}\text { Min. } \\
\text { Temp }\end{array}$ & $\begin{array}{l}\text { Max. } \\
\text { Temp }\end{array}$ & $\begin{array}{l}\text { Min. } \\
\text { Wind } \\
\end{array}$ & $\begin{array}{l}\text { Max. } \\
\text { Wind } \\
\end{array}$ & $\begin{array}{l}\text { Min. } \\
\text { Snow }\end{array}$ & $\begin{array}{l}\text { Max. } \\
\text { Snow } \\
\end{array}$ & AM Sky & PM Sky \\
\hline 44. LARONGE & -4 & -3 & 0 & 5 & 30 & 40 & partly cloudy & overcast \\
\hline $\begin{array}{l}\text { 45. LAST MOUNTAIN } \\
\text { LAKE N.W.A. }\end{array}$ & -16 & -12 & 20 & 40 & 0 & 5 & overcast & overcast \\
\hline 46. LEADER (North) & -24 & -8 & 0 & 10 & 5 & 80 & clear & clear \\
\hline 47. LEADER (South) & -24 & -7 & & 17 & 30 & 60 & $\begin{array}{l}\text { overcast; light fog } \\
\text { and snow }\end{array}$ & $\begin{array}{l}\text { overcast; } \\
\text { light snow }\end{array}$ \\
\hline 48. LIVELONG & -18 & -9 & 0 & 0 & & & overcast; light fog & clear \\
\hline $\begin{array}{l}\text { 49. LOVE - } \\
\text { TORCH RIVER }\end{array}$ & -18 & -10 & 0 & 0 & 40 & 60 & $\begin{array}{l}\text { overcast; } \\
\text { moderate fog }\end{array}$ & overcast; light fog \\
\hline 50. LUSELAND & -14 & -8 & 0 & 20 & 10 & 30 & $\begin{array}{l}\text { overcast; } \\
\text { light snow }\end{array}$ & $\begin{array}{l}\text { overcast; } \\
\text { light snow }\end{array}$ \\
\hline 51. MACDOWALL & -11 & -9 & 3 & 10 & 20 & 35 & $\begin{array}{l}\text { overcast; moder- } \\
\text { ate fog and snow }\end{array}$ & $\begin{array}{l}\text { overcast; moder- } \\
\text { ate fog and snow }\end{array}$ \\
\hline 52. MEADOW LAKE & -10 & -2 & 0 & 0 & & & & \\
\hline 53. MELFORT & -15 & -10 & 0 & 15 & 30 & 40 & partly cloudy & partly cloudy \\
\hline 54. MELFORT (SE) & -15 & -4 & 3 & 5 & 15 & 45 & $\begin{array}{l}\text { overcast; light fog; } \\
\text { heavy snow }\end{array}$ & $\begin{array}{l}\text { overcast; heavy } \\
\text { fog; heavy snow }\end{array}$ \\
\hline 55. MELVILLE & -3 & -6 & 0 & 10 & 5 & 90 & $\begin{array}{l}\text { overcast; } \\
\text { moderate fog }\end{array}$ & $\begin{array}{l}\text { overcast; } \\
\text { moderate fog }\end{array}$ \\
\hline 56. MISSINIPE & -10 & -4 & 0 & 0 & 32 & 32 & $\begin{array}{l}\text { overcast; } \\
\text { moderate snow }\end{array}$ & $\begin{array}{l}\text { overcast; } \\
\text { light snow }\end{array}$ \\
\hline 57. MOOSE JAW & -11 & -8 & 0 & 5 & & & clear & clear \\
\hline 58. MOOSE MOUNTAIN & -19 & -4 & 0 & 10 & 13 & 32 & clear & clear \\
\hline 59. NIPAWIN & -15 & -9 & 5 & 10 & 75 & 75 & & $\begin{array}{l}\text { overcast; } \\
\text { light snow }\end{array}$ \\
\hline $\begin{array}{l}\text { 60. PADDOCKWOOD - } \\
\text { CHRISTOPHER L. }\end{array}$ & -4 & -3 & 5 & 10 & 40 & 50 & overcast & overcast \\
\hline 61. PIKE LAKE & -18 & -10 & 4 & 15 & 2 & 20 & clear & clear \\
\hline 62. PRINCE ALBERT & -24 & -15 & 0 & 15 & 20 & 40 & overcast & partly cloudy \\
\hline $\begin{array}{l}\text { 63. PRINCE ALBERT } \\
\text { NATIONAL PARK } \\
\end{array}$ & -18 & -12 & 0 & 5 & 22 & 38 & overcast & overcast \\
\hline $\begin{array}{l}\text { 64. QU'APPELLE } \\
\text { VALLEY DAM }\end{array}$ & -17 & -12 & 0 & 15 & 1 & 60 & $\begin{array}{l}\text { overcast; } \\
\text { light snow }\end{array}$ & mostly clear \\
\hline 65. RAYMORE & -13 & -4 & 0 & 20 & 0 & 30 & clear & clear \\
\hline 66. REGINA & -15 & -8 & 5 & 28 & 20 & 70 & mostly clear & mostly clear \\
\hline $\begin{array}{l}\text { 67. ROCKGLEN - } \\
\text { BORDERLAND }\end{array}$ & -10 & -8 & 0 & 5 & 5 & 5 & mostly clear & partly cloudy \\
\hline $\begin{array}{l}\text { 68. ROUND LAKE } \\
\text { (Qu'Appelle Valley) }\end{array}$ & -19 & -14 & 0 & 5 & 10 & 35 & $\begin{array}{l}\text { overcast; } \\
\text { light snow }\end{array}$ & clear \\
\hline $\begin{array}{l}\text { 69. ROUND LAKE } \\
\text { (Prince Albert) }\end{array}$ & -18 & & & & 60 & 75 & & \\
\hline $\begin{array}{r}\text { 70. SASKATCHEWAN } \\
\text { LANDING P. PARK }\end{array}$ & -7 & -3 & 0 & 10 & 0 & 10 & mostly clear & partly cloudy \\
\hline 71. SASKATOON & -17 & -8 & 0 & 8 & 5 & 30 & clear & clear \\
\hline 72. SCOTT & -16 & -11 & 0 & 20 & 0 & 20 & mostly clear & partly cloudy \\
\hline 73. SHAMROCK & -7 & -7 & 0 & 5 & & & mostly cloudy & mostly cloudy \\
\hline 74. SHAUNAVON & -10 & -8 & 0 & 0 & 30 & 30 & clear & clear \\
\hline 75. SKULL CREEK & 1 & 2 & 0 & 0 & 0 & 22 & clear & clear \\
\hline 76. SNOWDEN & -14 & -9 & 0 & 0 & 20 & 33 & heavy fog & heavy fog \\
\hline 77. SPALDING & -5 & -5 & 0 & 0 & 10 & 100 & overcast & overcast \\
\hline 78. SPINNEY HILL & -13 & -6 & 0 & 10 & 0 & 30 & & \\
\hline 79. SQUAW RAPIDS & -10 & -5 & 30 & 40 & 15 & 18 & overcast & partly cloudy \\
\hline 80. SWIFT CURRENT & -16 & -12 & & 15 & & & clear & mostly clear \\
\hline 81. TISDALE & -15 & -10 & 10 & 12 & 40 & 50 & clear & partly cloudy \\
\hline 82. TURTLE LAKE & -20 & -14 & 0 & 0 & & 30 & clear & mostly clear \\
\hline 83. WEYBURN & -20 & -10 & 10 & 15 & 10 & 15 & overcast & overcast \\
\hline 84. WHITE BEAR & -10 & -10 & 0 & 0 & 10 & 60 & clear & clear \\
\hline 85. WHITEWOOD & -28 & -18 & & & 15 & 40 & overcast & overcast \\
\hline 86. YORKTON & & -12 & 0 & 5 & & & & clear \\
\hline
\end{tabular}




\begin{tabular}{|c|c|c|c|c|c|c|c|}
\hline & 高 & & & 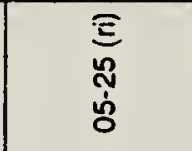 & 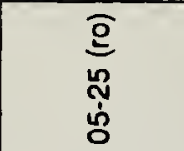 & $\begin{array}{l}\text { En } \\
\stackrel{n}{n} \\
\dot{n}\end{array}$ & 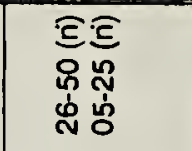 \\
\hline & 高 & & & 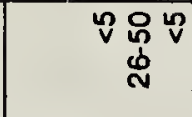 & 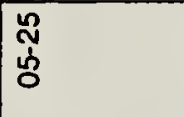 &  & 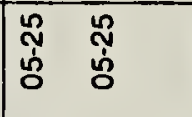 \\
\hline & 巳̃ &  & 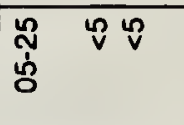 & 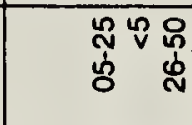 & งทำ &  & $\begin{array}{ll}\stackrel{2}{*} & \text { v } \\
\text { 官 } & \end{array}$ \\
\hline & 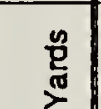 & 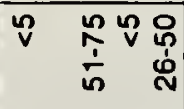 & 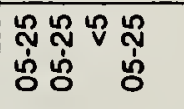 & 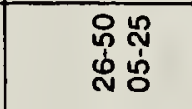 & 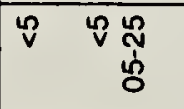 & 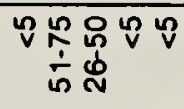 & 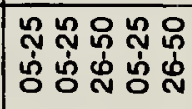 \\
\hline & 今े & 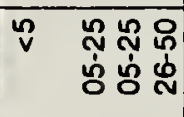 & 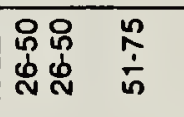 & 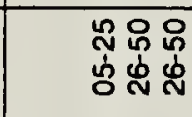 & ขั & 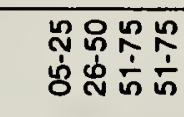 & 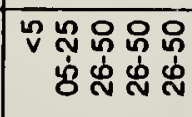 \\
\hline & Е웡 & & 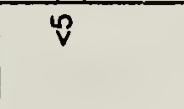 & 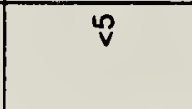 & & 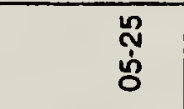 & 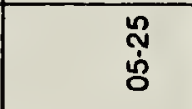 \\
\hline & 窢 & 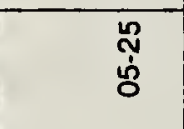 & จุ & 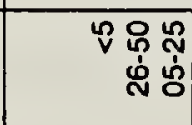 & $\vartheta$ & $\bar{v}$ & v \\
\hline & $\frac{9}{80}$ & 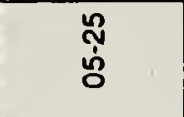 & 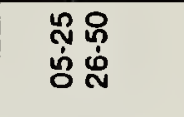 & 营 & 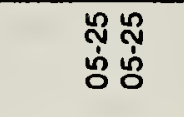 & & 实 \\
\hline & 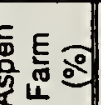 & 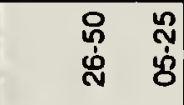 & 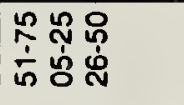 & 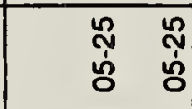 &  & $\bar{v}$ & 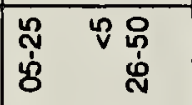 \\
\hline & 응 &  &  & $v$ & 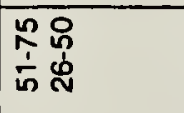 & $\begin{array}{l}\text { 员 } \\
\text { d }\end{array}$ & $\begin{array}{l}\text { O̊ } \\
\text { d. }\end{array}$ \\
\hline & ब。워 &  & $\begin{array}{l}\text { : } \\
\stackrel{\leftrightarrow}{\circ}\end{array}$ & $\vartheta$ & 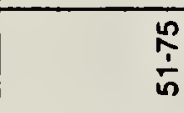 & $\underset{n}{n}$ & 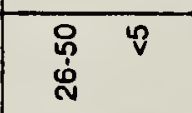 \\
\hline & 离 & $\bar{v}$ & $\begin{array}{ll}\stackrel{\leftrightarrow}{*} \\
\end{array}$ & $\sum_{\lambda}^{n} v$ & & & $\tilde{v}$ \\
\hline & 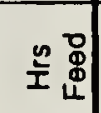 & & 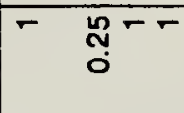 & फ़्Nल & $-\infty=$ & MNNO & $m$ \\
\hline & 农尔 & $\cong$ & $000 \dddot{00}$ & $\prod_{\infty}^{n} \mathbb{N}^{\infty}$ & 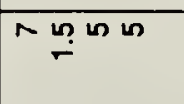 & लNO & 0 \\
\hline & కร 이 & $\stackrel{\leftrightarrow}{\varpi}$ & 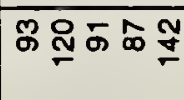 & 웅유 & $\frac{0}{2} 285$ & 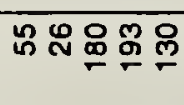 & 웜유 \\
\hline & 奌悹 & N & חִNO & $--\underset{\underline{m}}{n}-0$ & 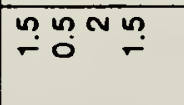 & 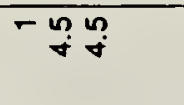 & $\underset{\sim \sim}{N} \sim \underset{\sim}{n} \infty$ \\
\hline & E & N & mN & $\stackrel{n}{m} \mathbb{N}$ & $--\theta N$ & ㄴ̣̣a & 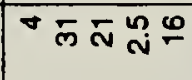 \\
\hline  & : & 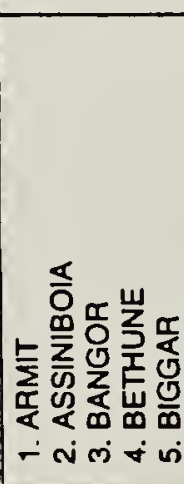 &  & 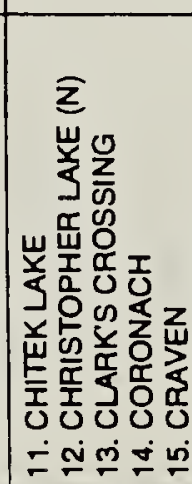 & 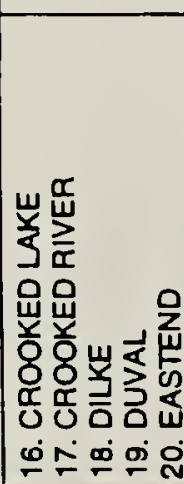 &  & 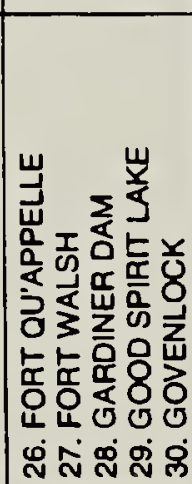 \\
\hline
\end{tabular}






\begin{tabular}{|c|c|c|c|c|c|c|c|}
\hline & 壱 ᄋ & & & & & & 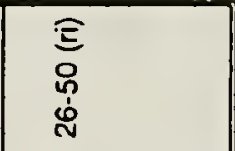 \\
\hline & 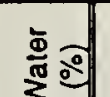 & & 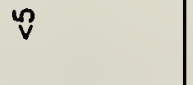 & $v$ & & & \\
\hline & อ๊ & 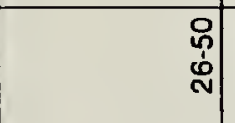 & 总 $\backsim v$ & 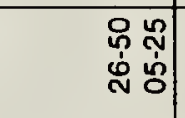 & 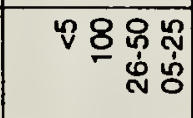 &  & 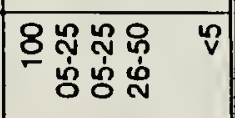 \\
\hline & 造 & 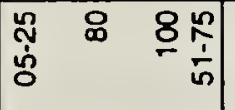 & 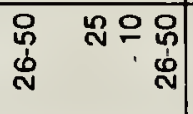 & 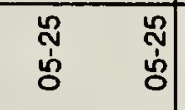 & 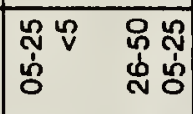 & 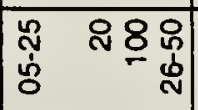 & 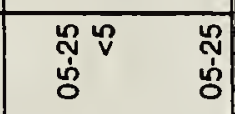 \\
\hline & 今े & 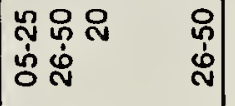 & 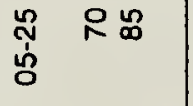 & 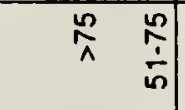 &  & \begin{tabular}{|cc|}
\multirow{2}{*}{} & \multirow{N}{*}{} \\
ஸे & $\dot{0}$ \\
\end{tabular} & 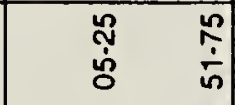 \\
\hline & 㝕 & & & $\begin{array}{l}0 \stackrel{\sim}{N} \\
\dot{b} \\
\dot{0}\end{array}$ & $\begin{array}{|ll|}0 & 0 \\
\dot{0} & \\
N & \\
\end{array}$ & & \\
\hline & : & 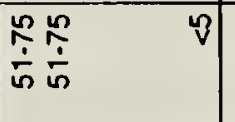 & & 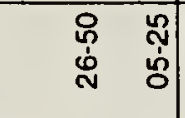 &  & & \\
\hline & 客题 & 恚 &  & & 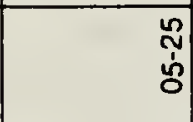 & 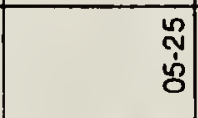 & 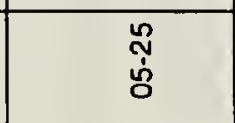 \\
\hline & 密 & & 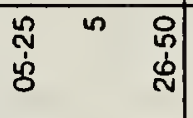 & $\begin{array}{l}\text { O } \\
\stackrel{0}{N}\end{array}$ &  & 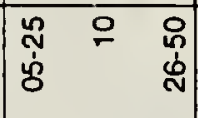 & 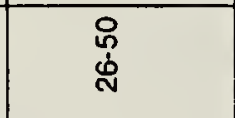 \\
\hline & 뭉 & &  & 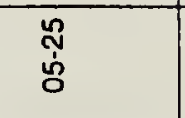 & & 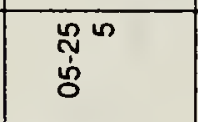 & 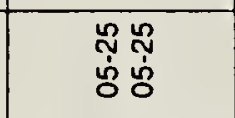 \\
\hline & $\frac{\mathrm{x}}{\Sigma} \mathrm{a}$ & & $\begin{array}{ll}\stackrel{0}{0} & v \\
\dot{\mathscr{d}} & \end{array}$ & 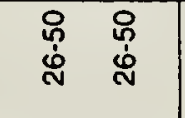 &  &  & $\begin{array}{ll}\stackrel{\mathscr{N}}{\tilde{H}} & \stackrel{\Lambda}{\Lambda} \\
\end{array}$ \\
\hline & 兽 & & \begin{tabular}{ll|}
\multirow{4}{*}{} & $v$ \\
రे & \\
\end{tabular} & 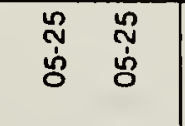 & $\stackrel{\varphi}{v}$ & \begin{tabular}{l}
\multirow{2}{*}{} \\
$\dot{H}$ \\
0
\end{tabular} & 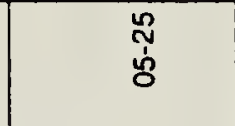 \\
\hline & 象竞 & $\nabla m 0$ & $N N$ & $\wedge \stackrel{0}{0}$ & $m \nabla \infty \mathbb{N}^{\sigma}$ & $\forall \nabla \% \infty N$ & का \\
\hline & 空 & कल- & $8+\infty 0$ & w० भुन & ar ma & mar- & Nor \\
\hline & 占 ơ & $\operatorname{lon}^{\circ}$ & ร8: & 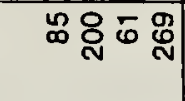 & q 绐气 & 웡응ㅇㅇㅛ & Фळ \\
\hline ఫ్d & 尘 & & $-n$ & $--\underline{n}-$ & $N \nabla$ & NNMO & Nִn \\
\hline $\begin{array}{l}0 \\
0 \\
0 \\
0\end{array}$ & 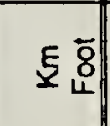 & का & N- & $n-a n$ & No & 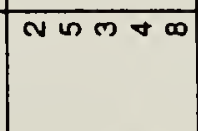 & $\Phi N$ \\
\hline 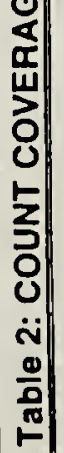 & : &  & 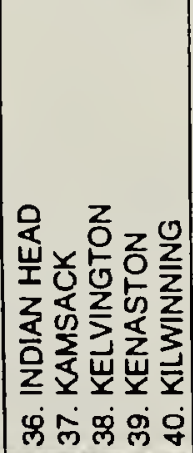 & 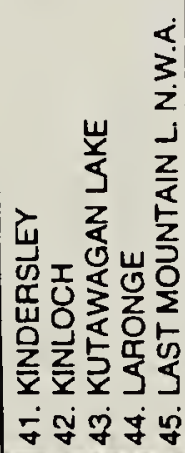 & 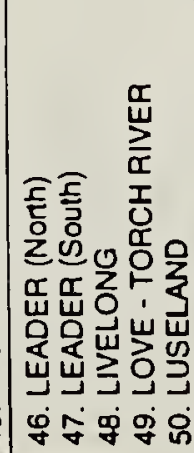 & 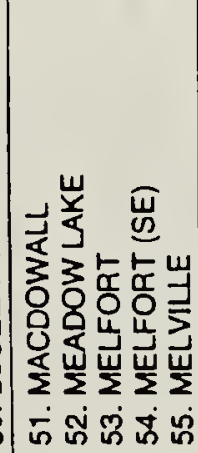 & 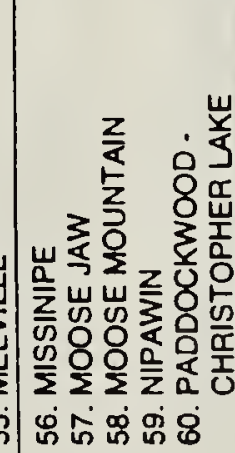 \\
\hline
\end{tabular}




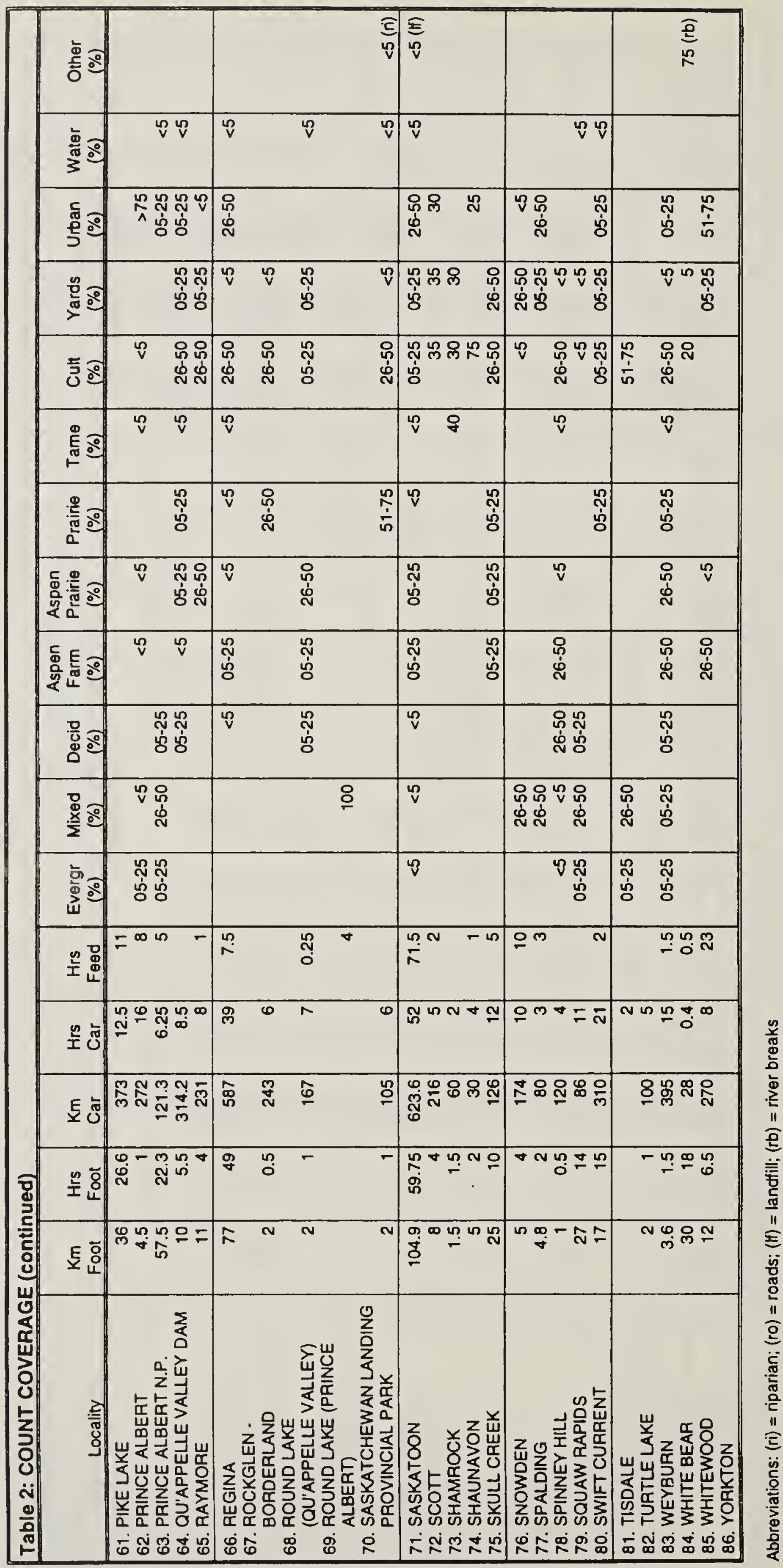


CANADA GOOSE

MALLARD

COMMON GOLDENEYE

BALD EAGLE

NORTHERN GOSHAWK

GOLDEN EAGLE

MERLIN

GRAY PARTRIDGE

RING-NECKED PHEASANT

RUFFED GROUSE

SHARP-TALLED GROUSE

ROCK DOVE

GREAT HORNED OWL

SNOWY OWL

DOWNY WOODPECKER

HAIRY WOODPECKER

NORTHERN FLICKER $(Y-s)$

PILEATED WOODPECKER

HORNED LARK

GRAY JAY

BLUE JAY

BLACK-BILLED MAGPIE

AMERICAN CROW

COMMON RAVEN

BLACK-CAPPED CHICKADEE

BOREAL CHICKADEE

RED-BREASTED NUTHATCH

WHITE-BREASTED NUTHATCH

AMERICAN ROBIN

BOHEMIAN WAXWING

CEDAR WAXWING

NORTHERN SHRIKE

EUROPEAN STARLING

DARK-EYED JUNCO

SNOW BUNTING

RUSTY BLACKBIRD

PINE GROSBEAK

PURPLE FINCH

HOUSE FINCH

WHITE-WINGED CROSSBILL

COMMON REDPOLL

HOARY REDPOLL

PINE SISKIN

EVENING GROSBEAK

HOUSE SPARROW

INDIVIDUALS IN TABLES 4 \& 5

SPECIES IN TABLES $4 \& 5$

NO. SPECIES COUNT DAY

NO. SPECIES COUNT PERIOD

NO. INDIVIDUALS COUNT DAY

\begin{tabular}{|c|c|c|c|c|c|c|c|c|c|}
\hline 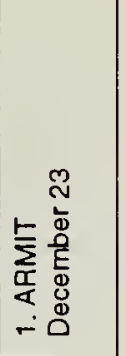 & 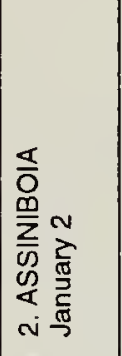 & 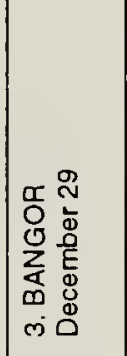 & 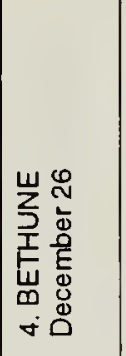 & 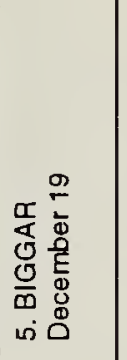 & 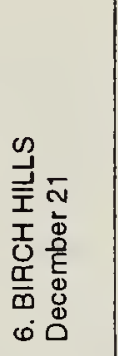 &  & 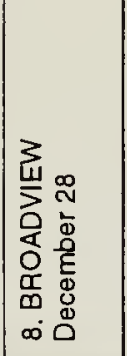 & 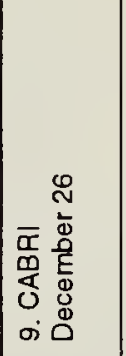 &  \\
\hline & & & & & & & & & \\
\hline & & & & & & & & & \\
\hline & & & & & & & & & \\
\hline & & & + & & & & & & \\
\hline & & & & 1 & + & & & & \\
\hline & & 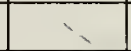 & & 1 & & & & + & \\
\hline & & & + & & 1 & & & & \\
\hline & 96 & + & + & 23 & 5 & & & & \\
\hline & 6 & & & & & & & & \\
\hline 5 & & 2 & & & 1 & & 1 & & \\
\hline 11 & 4 & 3 & 4 & 93 & & 11 & 8 & & \\
\hline & & 5 & 6 & 149 & 41 & 19 & 32 & 40 & \\
\hline & 4 & 1 & & 2 & 1 & & + & & \\
\hline & 1 & & + & & 1 & & 1 & 2 & \\
\hline & & 2 & & 3 & 5 & 2 & 3 & & 2 \\
\hline 1 & & 3 & & 3 & 3 & 2 & + & & 4 \\
\hline & & & 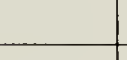 & & & & &  & \\
\hline & & & 1 & & ? & & & 8 & \\
\hline 6 & & & & 2 & 2 & & $y$ & 8 & 8 \\
\hline 6 & & 1 & & 1 & 5 & & 1 & & 4 \\
\hline 52 & 42 & 5 & 4 & 191 & 27 & $47 !$ & 34 & 7 & 11 \\
\hline & & & & & & & & & \\
\hline 75 & & 3 & & 28 & 4 & 1 & 9 & & 130 \\
\hline 20 & & 26 & 2 & 20 & 43 & 8 & 37 & & 29 \\
\hline 1 & & & & & & & & & 6 \\
\hline 22 & & 2 & & 1 & & 11 & & & 5 \\
\hline 1 & & 2 & & & & & 6 & & \\
\hline & & & & & & & & & \\
\hline & 4 & + & & 348 & 1 & 85 & & & \\
\hline & & & & & & & & & \\
\hline & & & & & & & & & \\
\hline & & & & 2 & & & & & \\
\hline & & 1 & & & & & & & \\
\hline 233 & 10 & 60 & 25 & 188 & 155 & 1 & 3 & 25 & \\
\hline & & & & & & & & & \\
\hline 17 & & 9 & & 19 & + & 5 & 5 & & 21 \\
\hline & & & & & & & & & \\
\hline 601 & & & & 7 & 6 & & 1 & & 40 \\
\hline 11 & & 14 & & 269 & 68 & 13 & 104 & & 50 \\
\hline & & & & & & & & & \\
\hline & & & & 2 & & & & & \\
\hline 91 & & & & & 23 & & & & 154 \\
\hline 51 & 350 & 11 & 50 & 680 & 195 & 14 & 32 & 60 & \\
\hline 9 & 0 & 0 & 0 & 2 & 0 & 0 & 1 & 1 & 3 \\
\hline 5 & 0 & 1 & 0 & 2 & 0 & 0 & 1 & 1 & 2 \\
\hline 22 & 9 & 17 & 6 & 24 & 19 & 13 & 17 & 7 & 15 \\
\hline 22 & 9 & 20 & 10 & 24 & 21 & 13 & 19 & 8 & 15 \\
\hline 710 & 517 & 150 & 91 & 2035 & 587 & 209 & 279 & 143 & 467 \\
\hline
\end{tabular}


Table 3-2: SPECIES RECORDED FROM MORE THAN SIX LOCALITIES $(t=$ species seen during the count period but not on count day)

\section{SPECIES}

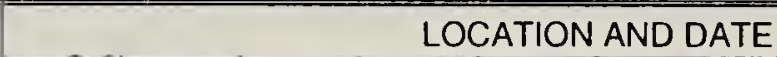

CANADA GOOSE

MALLARD

COMMON GOLDENEYE

BALD EAGLE

NORTHERN GOSHAWK

GOLDEN EAGLE

MERLIN

GRAY PARTRIDGE

RING-NECKED PHEASANT

RUFFED GROUSE

SHARP-TAILED GROUSE

ROCK DOVE

GREAT HORNED OWL

SNOWY OWL

DOWNY WOODPECKER

HAIRY WOODPECKER

NORTHERN FLICKER (Y-s)

PILEATED WOODPECKER

HORNED LARK

GRAY JAY

BLUE JAY

BLACK-BILLED MAGPIE

AMERICAN CROW

COMMON RAVEN

BLACK-CAPPED CHICKADEE

BOREAL CHICKADEE

RED-BREASTED NUTHATCH

WHITE-BREASTED NUTHATCH

AMERICAN ROBIN

BOHEMIAN WAXWING

CEDAR WAXWING

NORTHERN SHRIKE

EUROPEAN STARLING

DARK-EYED JUNCO

SNOW BUNTING

RUSTY BLACKBIRD

PINE GROSBEAK

PURPLE FINCH

HOUSE FINCH

WHITE-WINGED CROSSBILL

COMMON REDPOLL

HOARY REDPOLL

PINE SISKIN

EVENING GROSBEAK

HOUSE SPARROW



LOCATION AND DATE



INDIVIDUALS IN TABLES 4 \& 5

SPECIES IN TABLES $4 \& 5$

NO. SPECIES COUNT DAY

NO. SPECIES COUNT PERIOD

NO. INDIVIDUALSS COUNT DAY

\begin{tabular}{|l}
\hline \\
\hline \\
\hline
\end{tabular}

5448

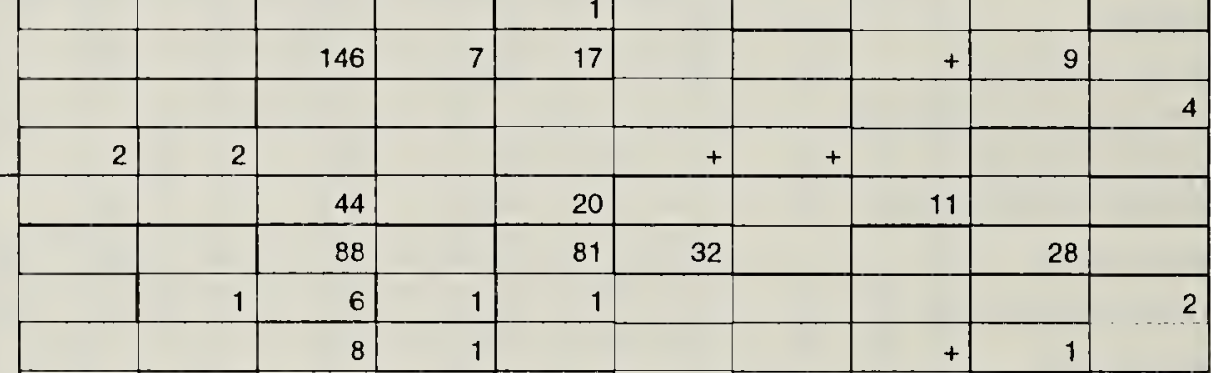

\begin{tabular}{|r|r|r|r|r|r|r|r|r|r|}
\hline 1 & 2 & 3 & & 5 & 4 & 2 & & 3 & 6 \\
\hline & 2 & 6 & & 9 & 5 & 2 & & 2 & 8 \\
\hline & & & & & & & & & \\
\hline & + & & & & & & & & \\
\hline & & 1 & 1590 & & & & & & \\
\hline
\end{tabular}




\begin{tabular}{|c|c|c|c|c|c|c|c|c|c|c|}
\hline $\begin{array}{l}\text { Table 3-3: SPECIES RE } \\
\text { (+ = species seen durir }\end{array}$ & $\begin{array}{l}\text { ED F } \\
\text { coun }\end{array}$ & ROM N & $\begin{array}{l}\text { MORE } \\
\text { d but } r\end{array}$ & $\begin{array}{l}\text { THAN } \\
\text { pot on }\end{array}$ & $\begin{array}{l}\text { SIX LO } \\
\text { count }\end{array}$ & $\begin{array}{l}\text { CALIT } \\
\text { day) }\end{array}$ & & & & \\
\hline & & & & $\mathrm{LOC}$ & CATION & AND D & ATE & & & \\
\hline SPECIES & 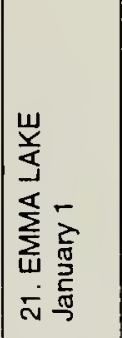 & 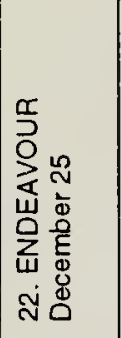 & 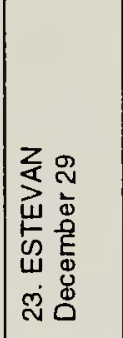 &  & 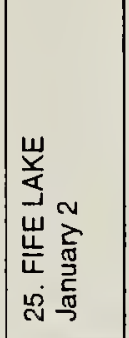 & 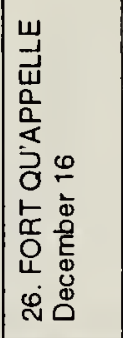 & 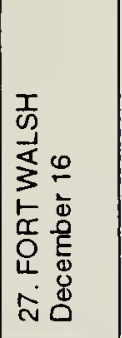 & 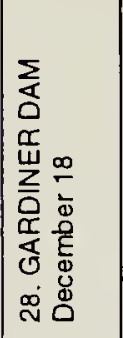 & 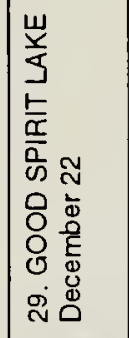 & 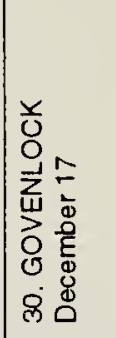 \\
\hline CANADA GOOSE & & & + & & 679 & + & & & & \\
\hline MALLARD & & & 269 & & 2500 & 5 & 1 & 3878 & & 1 \\
\hline COMMON GOLDENEYE & & & 16 & & 5 & 13 & & 65 & & \\
\hline BALD EAGLE & & & 2 & & & 1 & 5 & 11 & & 1 \\
\hline NORTHERN GOSHAWK & & & & & & 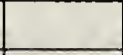 & 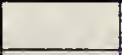 & 2 & + & \\
\hline GOLDEN EAGLE & & & 1 & & 1 & + & + & 2 & & 1 \\
\hline MERLIN & & & & & & & & & & \\
\hline GRAY PARTRIDGE & & & & & 18 & & 16 & 121 & 8 & 38 \\
\hline RING-NECKED PHEASANT & & & 2 & & 1 & + & 4 & & & 3 \\
\hline RUFFED GROUSE & 1 & 2 & & & 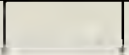 & 3 & 1 & & 2 & \\
\hline SHARP-TAILED GROUSE & & & & & 74 & 18 & 59 & 65 & 13 & 30 \\
\hline ROCK DOVE & & & 57 & 5 & 30 & + & 1 & 64 & 3 & 11 \\
\hline GREAT HORNED OWL & & & 2 & + & & + & 1 & 2 & + & 6 \\
\hline SNOWY OWL & & & & & & & & 1 & & 4 \\
\hline DOWNY WOODPECKER & 2 & & 11 & 1 & & 5 & 2 & 1 & 10 & \\
\hline HAIRY WOODPECKER & 3 & 1 & 3 & 5 & & 8 & 7 & & 11 & \\
\hline NORTHERN FLICKER ( $Y$-s) & & & & & & 1 & & & & \\
\hline PILEATED WOODPECKER & + & & & & & & & & 1 & \\
\hline HORNED LARK & & & 22 & & 729 & & 11 & 11 & & 453 \\
\hline GRAY JAY & 2 & 3 & & & & + & & & & \\
\hline BLUE JAY & 21 & & 2 & 1 & & 22 & & 1 & 6 & \\
\hline BLACK-BILLED MAGPIE & & 8 & 15 & 12 & 5 & 52 & 163 & 138 & 34 & 8 \\
\hline AMERICAN CROW & & & & & & + & & & & \\
\hline COMMON RAVEN & 78 & 50 & & 4 & & 6 & 1 & 1 & 5 & \\
\hline BLACK-CAPPED CHICKADEE & 40 & 7 & 39 & 13 & & 169 & 66 & 3 & 53 & \\
\hline BOREAL CHICKADEE & 13 & & & & & & & & & \\
\hline RED-BREASTED NUTHATCH & 9 & & 3 & & & 5 & 12 & 3 & 1 & \\
\hline WHITE-BREASTED NUTHATCH & 5 & 1 & 14 & & & 11 & & & 3 & \\
\hline AMERICAN ROBIN & & & 3 & & & + & & & & \\
\hline BOHEMIAN WAXWING & & & 12 & & & 16 & 67 & 433 & & \\
\hline CEDAR WAXWING & & & & & & + & & & & \\
\hline NORTHERN SHRIKE & & & & & & & 4 & & 1 & \\
\hline EUROPEAN STARLING & & & + & & 3 & 6 & 1 & 14 & 2 & \\
\hline DARK-EYED JUNCO & & & & & & + & & & & \\
\hline SNOW BUNTING & 13 & 125 & 35 & 29 & 1500 & 62 & 104 & 66 & 186 & 480 \\
\hline RUSTY BLACKBIRD & & & & & & 6 & 8 & & & 2 \\
\hline PINE GROSBEAK & 34 & 23 & & & & 30 & 2 & 5 & 48 & \\
\hline PURPLE FINCH & & & & & & & & & & \\
\hline HOUSE FINCH & & & + & & & & & & & \\
\hline WHITE-WINGED CROSSBILL & & & 3 & & & + & 15 & & 2 & \\
\hline COMMON REDPOLL & + & 200 & 173 & 67 & 62 & 42 & 92 & 39 & 32 & 11 \\
\hline HOARY REDPOLL & & 7 & & & & + & & & + & \\
\hline PINE SISKIN & & & 46 & & & 30 & 3 & & & \\
\hline EVENING GROSBEAK & 40 & 20 & & 16 & & + & & & 11 & \\
\hline HOUSE SPARROW & 1 & 6 & 162 & & & 220 & 114 & 680 & 48 & 242 \\
\hline INDIVIDUALS IN TABLES $4 \& 5$ & 0 & 0 & 42 & 0 & 4200 & 2 & 84 & 439 & 0 & 24 \\
\hline SPECIES IN TABLES 4 \& 5 & 2 & 0 & 6 & $c$ & 1 & 5 & 9 & 2 & 0 & 4 \\
\hline NO. SPECIES COUNT DAY & 13 & 13 & 28 & 10 & 14 & 24 & 34 & 25 & 21 & 20 \\
\hline NO. SPECIES COUNT PERIOD & 17 & 13 & 31 & 11 & 14 & 39 & 35 & 25 & 24 & 20 \\
\hline NO. INDIVIDUALS COUNT DAY & 262 & 453 & 934 & 153 & 9807 & 733 & 844 & 6045 & 480 & 1315 \\
\hline
\end{tabular}









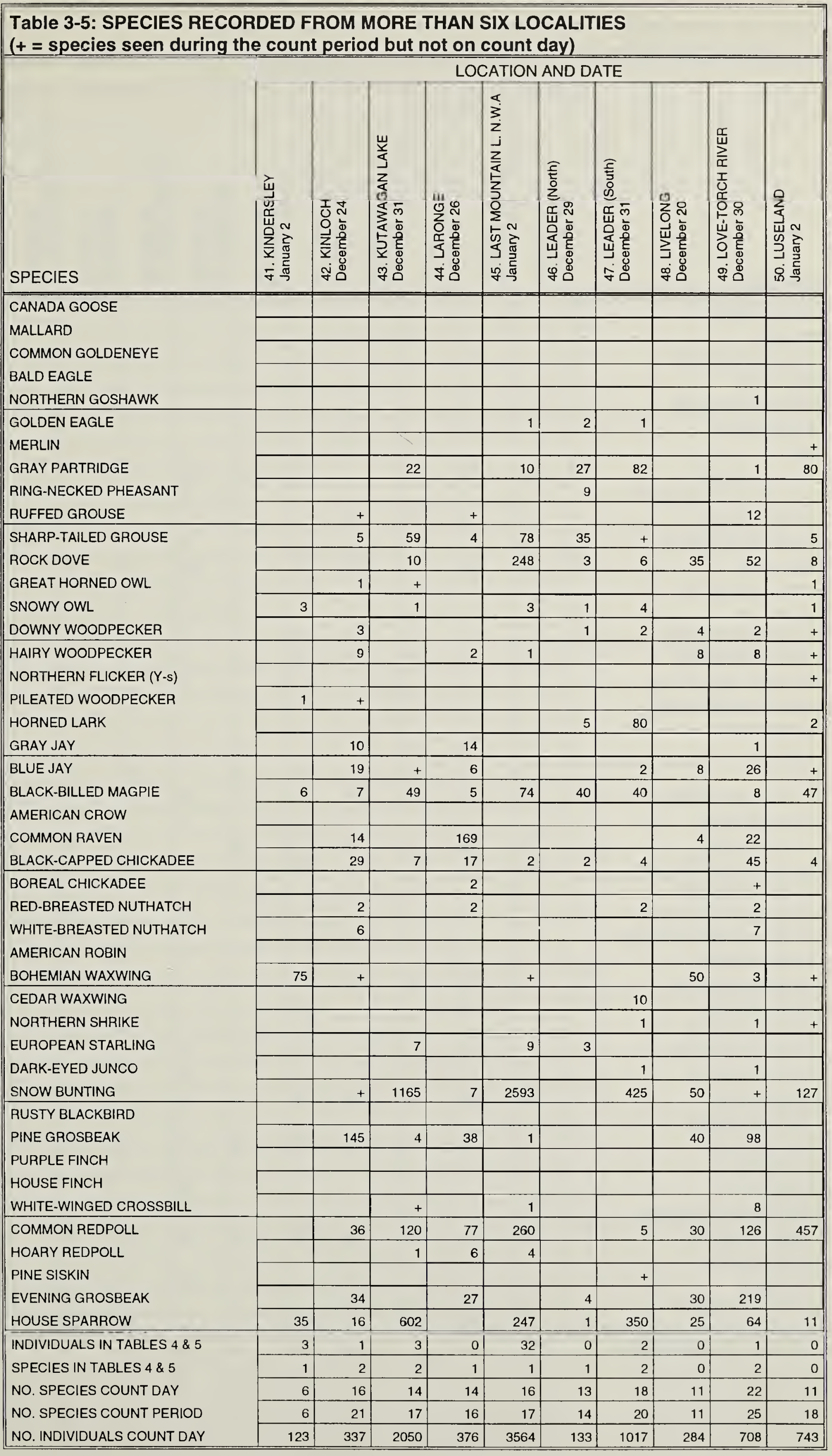




\section{Table 3-6: SPECIES RECORDED FROM MORE THAN SIX LOCALITIES}

$(+=$ species seen during the count period but not on count day)

\section{SPECIES}

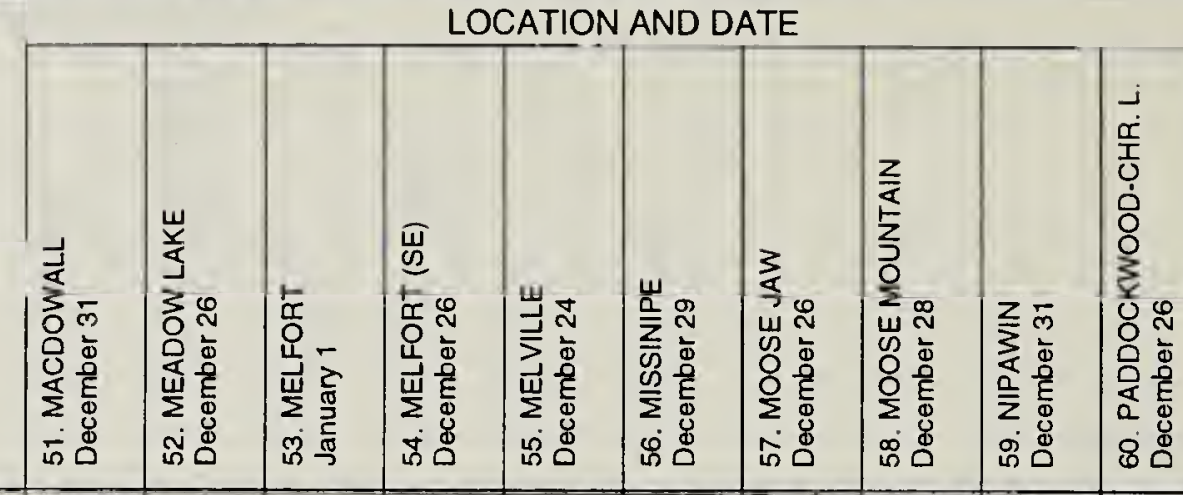

CANADA GOOSE

MALLARD

COMMON GOLDENEYE

BALD EAGLE

NORTHERN GOSHAWK

GOLDEN EAGLE

MERLIN

GRAY PARTRIDGE

RING-NECKED PHEASANT

RUFFED GROUSE

SHARP-TAILED GROUSE

ROCK DOVE

GREAT HORNED OWL

SNOWY OWL

DOWNY WOODPECKER

HAIRY WOODPECKER

NORTHERN FLICKER ( $Y$-s)

PILEATED WOODPECKER

HORNED LARK

GRAY JAY

BLUE JAY

BLACK-BILLED MAGPIE

AMERICAN CROW

COMMON RAVEN

BLACK-CAPPED CHICKADEE

BOREAL CHICKADEE

RED-BREASTED NUTHATCH

WHITE-BREASTED NUTHATCH

AMERICAN ROBIN

BOHEMIAN WAXWING

CEDAR WAXWING

NORTHERN SHRIKE

EUROPEAN STARLING

DARK-EYED JUNCO

SNOW BUNTING

RUSTY BLACKBIRD

PINE GROSBEAK

PURPLE FINCH

HOUSE FINCH

WHITE-WINGED CROSSBILL COMMON REDPOLL

HOARY REDPOLL

PINE SISKIN

EVENING GROSBEAK

HOUSE SPARROW

INDIVIDUALS IN TABLES $4 \& 5$

SPECIES IN TABLES 4 \& 5

NO. SPECIES COUNT DAY

NO. SPECIES COUNT PERIOD

NO. INDIVIDUALS COUNT DAY

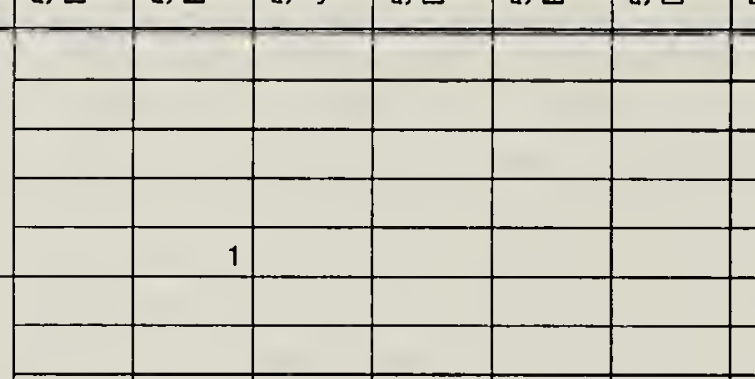

\begin{tabular}{l|l|l|l} 
& & \\
\hline & &
\end{tabular}




Table 3-7: SPECIES RECORDED FROM MORE THAN SIX LOCALITIES

$(+=$ species seen during the count period but not on count day)

SPECIES

CANADA GOOSE

MALLARD

COMMON GOLDENEYE

BALD EAGLE

NORTHERN GOSHAWK

GOLDEN EAGLE

MERLIN

GRAY PARTRIDGE

RING-NECKED PHEASANT

RUFFED GROUSE

SHARP-TAILED GROUSE

ROCK DOVE

GREAT HORNED OWL

SNOWY OWL

DOWNY WOODPECKER

HAIRY WOODPECKER

NORTHERN FLICKER (Y-s)

PILEATED WOODPECKER

HORNED LARK

GRAY JAY

BLUE JAY

BLACK·BILLED MAGPIE

AMERICAN CROW

COMMON RAVEN

BLACK-CAPPED CHICKADEE

BOREAL CHICKADEE

RED-BREASTED NUTHATCH

WHITE-BREASTED NUTHATCH

AMERICAN ROBIN

BOHEMIAN WAXWING

CEDAR WAXWING

NORTHERN SHRIKE

EUROPEAN STARLING

DARK-EYED JUNCO

SNOW BUNTING

RUSTY BLACKBIRD

PINE GROSBEAK

PURPLE FINCH

HOUSE FINCH

WHITE-WINGED CROSSBILL

COMMON REDPOLL

HOARY REDPOLL

PINE SISKIN

EVENING GROSBEAK

HOUSE SPARROW

INDIVIDUALS IN TABLES 4 \& 5

SPECIES IN TABLES 4 \& 5

NO. SPECIES COUNT DAY

NO. SPECIES COUNT PERIOD

NO. INDIVIDUALS COUNT DAY

\begin{tabular}{|c|c|c|c|c|c|c|c|c|c|}
\hline \multicolumn{10}{|c|}{ LOCATION AND DATE } \\
\hline \multirow[t]{3}{*}{ 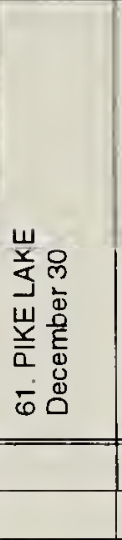 } & 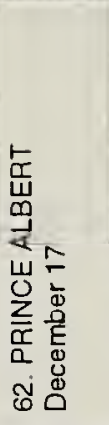 & 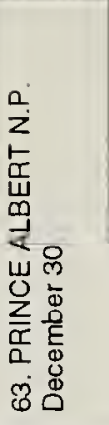 & 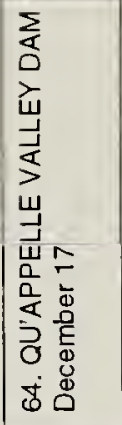 & 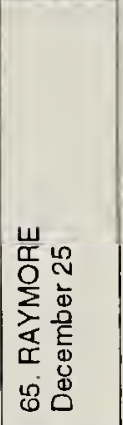 & 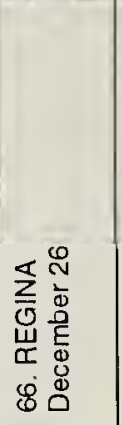 & 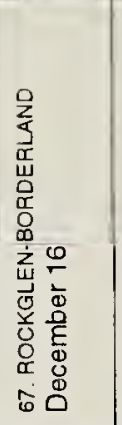 & 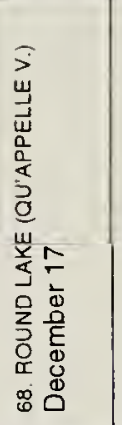 & 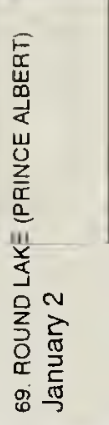 & 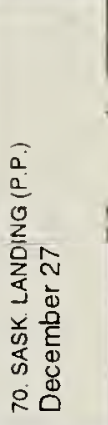 \\
\hline & & & & & 165 & & 2 & & \\
\hline & & & & & 66 & & & & \\
\hline 2 & & & 6 & & 1 & & & & \\
\hline 2 & & & & & & & 1 & & 1 \\
\hline \multirow[t]{3}{*}{1} & & & & & & & & & \\
\hline & & & & & & 2 & 1 & & 1 \\
\hline & & $y$ & & & 1 & 1 & & & \\
\hline \multirow[t]{2}{*}{4} & & & 59 & + & 105 & 44 & 14 & & 127 \\
\hline & & & & & & 22 & & & \\
\hline+ & 4 & 12 & & 2 & & & & & 1 \\
\hline 5 & & & 65 & 22 & 4 & 162 & + & + & 2 \\
\hline 25 & 433 & & 11 & 31 & 1002 & & 8 & & 23 \\
\hline \multirow[t]{2}{*}{4} & + & & 5 & 1 & 1 & 1 & + & & \\
\hline & & & 1 & & 10 & + & & & 1 \\
\hline 6 & 8 & 2 & 1 & 1 & 13 & & 9 & 2 & 2 \\
\hline 24 & 7 & 5 & 2 & 2 & 8 & & 9 & 5 & \\
\hline \multirow[t]{4}{*}{2} & & & & & 1 & & & & \\
\hline & & 2 & & & & & & & \\
\hline & & & & & & 298 & & & 2 \\
\hline & 5 & 25 & & & & & & 1 & \\
\hline
\end{tabular}

\begin{tabular}{|r|r|r|r|r|r|r|r|r|r|}
\hline 36 & 14 & 4 & & + & & & 12 & 12 & \\
\hline 194 & 53 & 17 & 85 & 57 & 176 & 14 & 27 & & 78 \\
\hline & 1 & & & & 1 & & & & \\
\hline 43 & 142 & 17 & 3 & 8 & & & 9 & 2 & \\
\hline 259 & 39 & 52 & 19 & 19 & 34 & & 119 & 30 & 4 \\
\hline
\end{tabular}

\begin{tabular}{|r|r|r|r|r|r|r|r|r|r|}
\hline 259 & 39 & 52 & 19 & 19 & 34 & & 119 & 30 & 4 \\
\hline & & 43 & & & & & & & \\
\hline & 5 & 6 & & + & 117 & & 3 & & \\
\hline 7 & 11 & 4 & & & 9 & & 10 & 2 & \\
\hline 188 & 87 & & & & 2 & & 1 & & \\
\hline
\end{tabular}

\begin{tabular}{|r|r|r|r|r|r|r|r|r|r|}
\hline 188 & 87 & & 92 & + & 263 & & & & + \\
\hline 2 & & & & & 4 & & & & \\
\hline 80 & & & 32 & 1 & 6 & + & 1 & & \\
\hline+ & 28 & & & + & 34 & & & & \\
\hline 41 & 44 & & 1 & 131 & 110 & & + & & \\
\hline
\end{tabular}


Table 3-8: SPECIES RECORDED FROM MORE THAN SIX LOCALITIES

$(t=$ species seen during the count period but not on count day)

LOCATION AND DATE

SPECIES

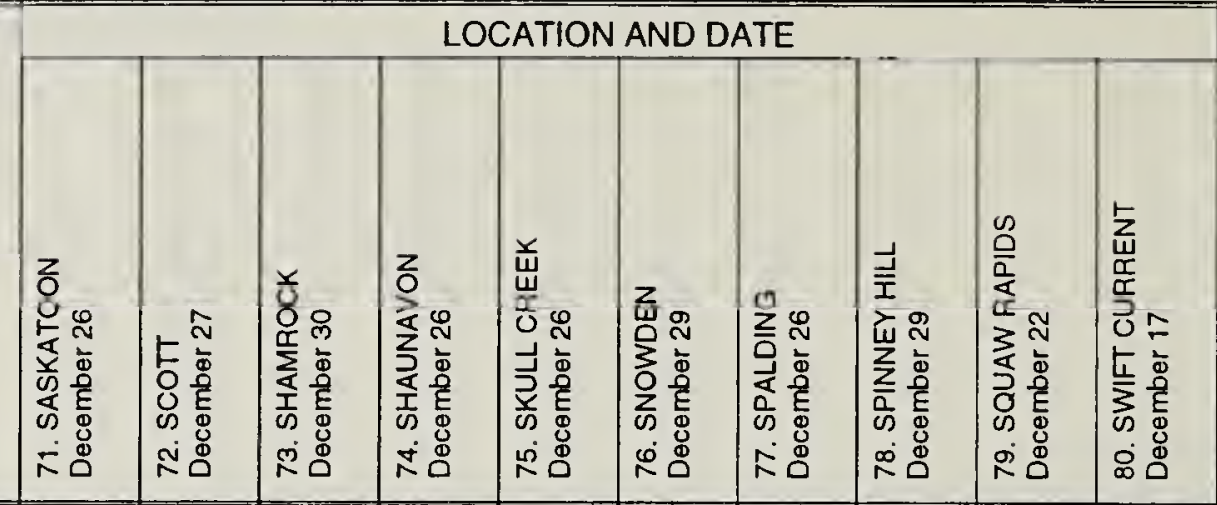

CANADA GOOSE

MALLARD

COMMON GOLDENEYE

BALD EAGLE

NORTHEAN GOSHAWK

GOLDEN EAGLE

MERLIN

GRAY PARTRIDGE

RING-NECKED PHEASANT

RUFFED GROUSE

SHARP-TAILED GROUSE

ROCK DOVE

GREAT HORNED OWL

SNOWY OWL

DOWNY WOODPECKER

HAIRY WOODPECKER

NORTHERN FLICKER $(Y-s)$

PILEATED WOODPECKER

HORNED LARK

GRAY JAY

BLUE JAY

BLACK-BILLED MAGPIE

AMERICAN CROW

COMMON RAVEN

BLACK-CAPPED CHICKADEE

BOREAL CHICKADEE

RED-BREASTED NUTHATCH

WHITE-BREASTED NUTHATCH

AMERICAN ROBIN

BOHEMIAN WAXWING

CEDAR WAXWING

NORTHERN SHRIKE

EUROPEAN STARLING

DARK.EYED JUNCO

SNOW BUNTING

RUSTY BLACKBIRD

PINE GROSBEAK

PURPLE FINCH

HOUSE FINCH

WHITE-WINGED CROSSBILL

COMMON REDPOLL

HOARY REDPOLL

PINE SISKIN

EVENING GROSBEAK

HOUSE SPARROW

INDIVIDUALS IN TABLES $4 \& 5$

SPECIES IN TABLES 4 \& 5

NO. SPECIES COUNT DAY

NO. SPECIES COUNT PERIOD

NO. INDIVIDUALS COUNT DAY

\begin{tabular}{|r|l|l|l|l|}
\hline 11 & & & & \\
\hline 230 & & & & \\
\hline & & & & \\
\hline & & & & \\
& & & &
\end{tabular}

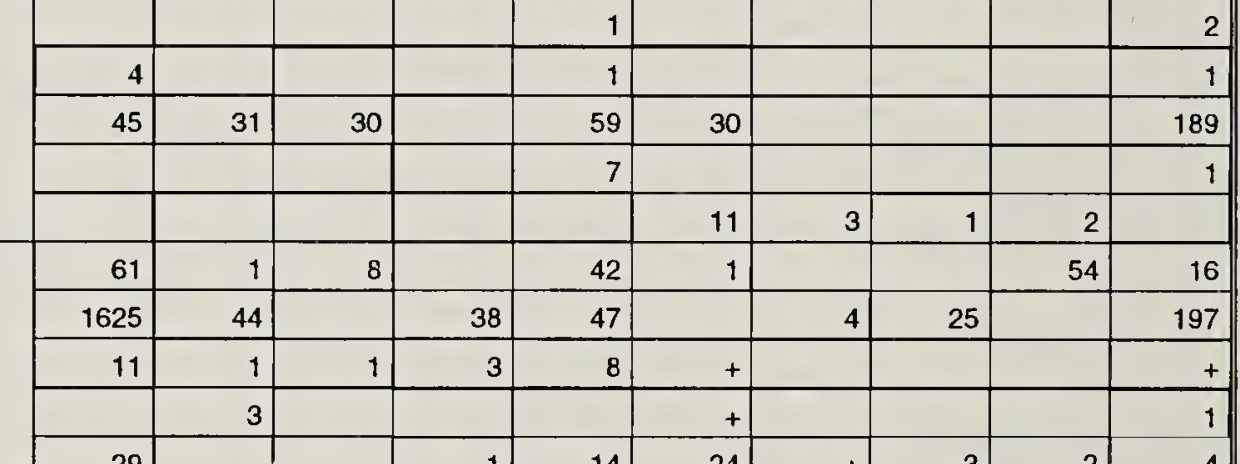

\begin{tabular}{|r|r|r|r|r|r|r|r|r|r||}
\hline 29 & & & 1 & 14 & 24 & + & 3 & 2 & 4 \\
\hline 33 & 1 & & & 18 & 22 & + & 2 & 3 & 3 \\
\hline 25 & & & & 3 & & & & & \\
\hline & & & & & + & & & 2 & \\
\hline 3 & & 33 & 15 & 261 & & & & & 6 \\
\hline & & & & & 17 & & & 18 & \\
\hline 111 & 2 & & & 3 & 25 & & 8 & 11 & \\
\hline 670 & 61 & 3 & 29 & 133 & 35 & 13 & 17 & 23 & 84 \\
\hline 5 & & & & & & & & & \\
\hline
\end{tabular}


Table 3-9: SPECIES RECORDED FROM MORE THAN SIX LOCALITIES

( $+=$ species seen during the count period but not on count day)

SPECIES

CANADA GOOSE

MALLARD

COMMON GOLDENEYE

BALD EAGLE

NORTHERN GOSHAWK

GOLDEN EAGLE

MERLIN

GRAY PARTRIDGE

RING-NECKED PHEASANT

RUFFED GROUSE

SHARP-TAILED GROUSE

ROCK DOVE

GREAT HORNED OWL

SNOWY OWL

DOWNY WOODPECKER

HAIRY WOODPECKER

NORTHERN FLICKER (Y-s)

PILEATED WOODPECKER

HORNED LARK

GRAY JAY

BLUE JAY

BLACK-BILLED MAGPIE

AMERICAN CROW

COMMON RAVEN

BLACK-CAPPED CHICKADEE

BOREAL CHICKADEE

RED-BREASTED NUTHATCH

WHITE-BREASTED NUTHATCH

AMERICAN ROBIN

BOHEMIAN WAXWING

CEDAR WAXWING

NORTHERN SHRIKE

EUROPEAN STARLING

DARK-EYED JUNCO

SNOW BUNTING

RUSTY BLACKBIRD

PINE GROSBEAK

PURPLE FINCH

HOUSE FINCH

WHITE-WINGED CROSSBILL

COMMON REDPOLL

HOARY REDPOLL

PINE SISKIN

EVENING GROSBEAK

HOUSE SPARROW

INDIVIDUALS IN TABLES 4 \& 5

SPECIES IN TABLES 4 \& 5

NO. SPECIES COUNT DAY

NO. SPECIES COUNT PERIOD

NO. INDIVIDUALS COUNT DAY

\begin{tabular}{|c|c|c|c|c|c|c|c|c|}
\hline \multicolumn{9}{|c|}{ LOCATION AND DATE } \\
\hline 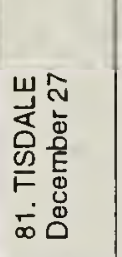 & 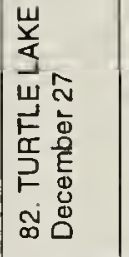 & 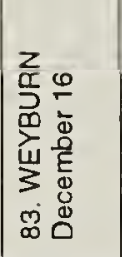 & 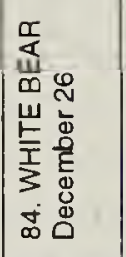 & 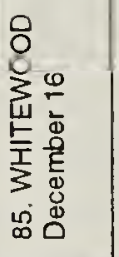 &  & 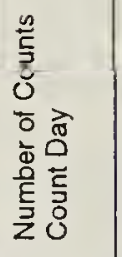 & 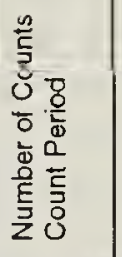 & 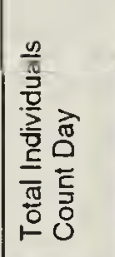 \\
\hline & & & & & & 7 & 9 & 1004 \\
\hline & & & & & 6 & 13 & 13 & 12217 \\
\hline & & & & & & 13 & 13 & 620 \\
\hline & & & & 1 & & 12. & 13 & 33 \\
\hline & & & + & & & 11 & 15 & 13 \\
\hline & & 2 & 4 & & & 19 & 22. & 31 \\
\hline & & + & + & 1 & 1 & 10 & 13 & 13 \\
\hline & & 246 & 69 & + & & 42 & 48 & 1978 \\
\hline & & 36 & + & & & 15 & 17 & 120 \\
\hline & 1 & & & 2 & & 27 & 33 & 109 \\
\hline & & 93 & + & 46 & & 45 & 50 & 1334 \\
\hline & 5 & 151 & 175 & 76 & 104 & 52 & 54 & 5996 \\
\hline 1 & & 6 & & 1 & & 35 & 45 & 87 \\
\hline & & 6 & + & & & 23 & 30 & 58 \\
\hline 1 & 3 & 1 & + & 13 & 1 & 63 & 66 & 307 \\
\hline & 1 & 1 & & 11 & 3 & 59 & 62 & 339 \\
\hline & & + & & & & 6 & 8 & 33 \\
\hline & & & & & 1 & 7 & 12 & 16 \\
\hline & & 3 & 2 & & & 29 & 29 & 3612 \\
\hline 1 & 5 & & & & & 25 & 26 & 152 \\
\hline 2 & 37. & & & 3 & 2 & 52 & 55 & 635 \\
\hline 2 & 21 & 51 & 61 & 82 & 17 & 78 & 78 & 3943 \\
\hline & & & & & & 6 & 8 & 16 \\
\hline 4. & 19 & & & 14 & 14 & 53 & 54 & 1408 \\
\hline 6 & 48 & 6 & & 269 & 48 & 73 & 73 & 3411 \\
\hline & 7 & & & & & 11 & 12 & 111 \\
\hline & 3 & 5 & & 12 & 9 & 42 & 44 & 660 \\
\hline & 6 & 2 & & 3 & 2 & 33 & 33 & 198 \\
\hline & & & & & 1 & 10 & 14 & 72 \\
\hline & & + & 30 & 47 & 211 & 38 & 46 & 5040 \\
\hline & & & & & & 6 & 7 & 267 \\
\hline & & + & & + & & 12 & 15 & 20 \\
\hline & & 39 & 5 & 13 & & 27 & 29 & 601 \\
\hline & & 3 & & + & 1 & 21 & 26 & 438 \\
\hline & & 400 & 45 & 86 & & 59 & 62 & 16021 \\
\hline & & 1 & & 2 & & 9 & 10 & 54 \\
\hline & 86 & & & 2 & 52 & 56 & 57 & 1939 \\
\hline & & 4 & & + & & 5 & 7 & 19 \\
\hline & & 14 & & + & & 5 & 7 & 143 \\
\hline & 4 & + & + & + & 70 & 31 & 38 & 1384 \\
\hline & 66 & 160 & + & 130 & 203 & 70 & 74 & 7902 \\
\hline & & 3 & & 11 & 5 & 20 & 22 & 122 \\
\hline & 1 & 17 & & 11 & & 25 & 27 & 906 \\
\hline & 33 & & & + & & 38 & 41 & 2194 \\
\hline 6 & 22 & 604 & 250 & 465 & 175 & 73 & 73 & 16919 \\
\hline 0 & 8 & 4 & 1 & 1 & 1. & & & \\
\hline 0 & 3. & 4 & 4 & 2 & 1 & & & \\
\hline 8 & 21 & 27 & 10 & 24 & 21 & & & \\
\hline 8 & 21 & 33 & 21 & 32 & 21 & & & \\
\hline 23 & 376 & 1858 & 642 & 1302 & 927 & & & \\
\hline
\end{tabular}


Table 4: SPECIES SEEN ON SIX OR FEWER COUNTS

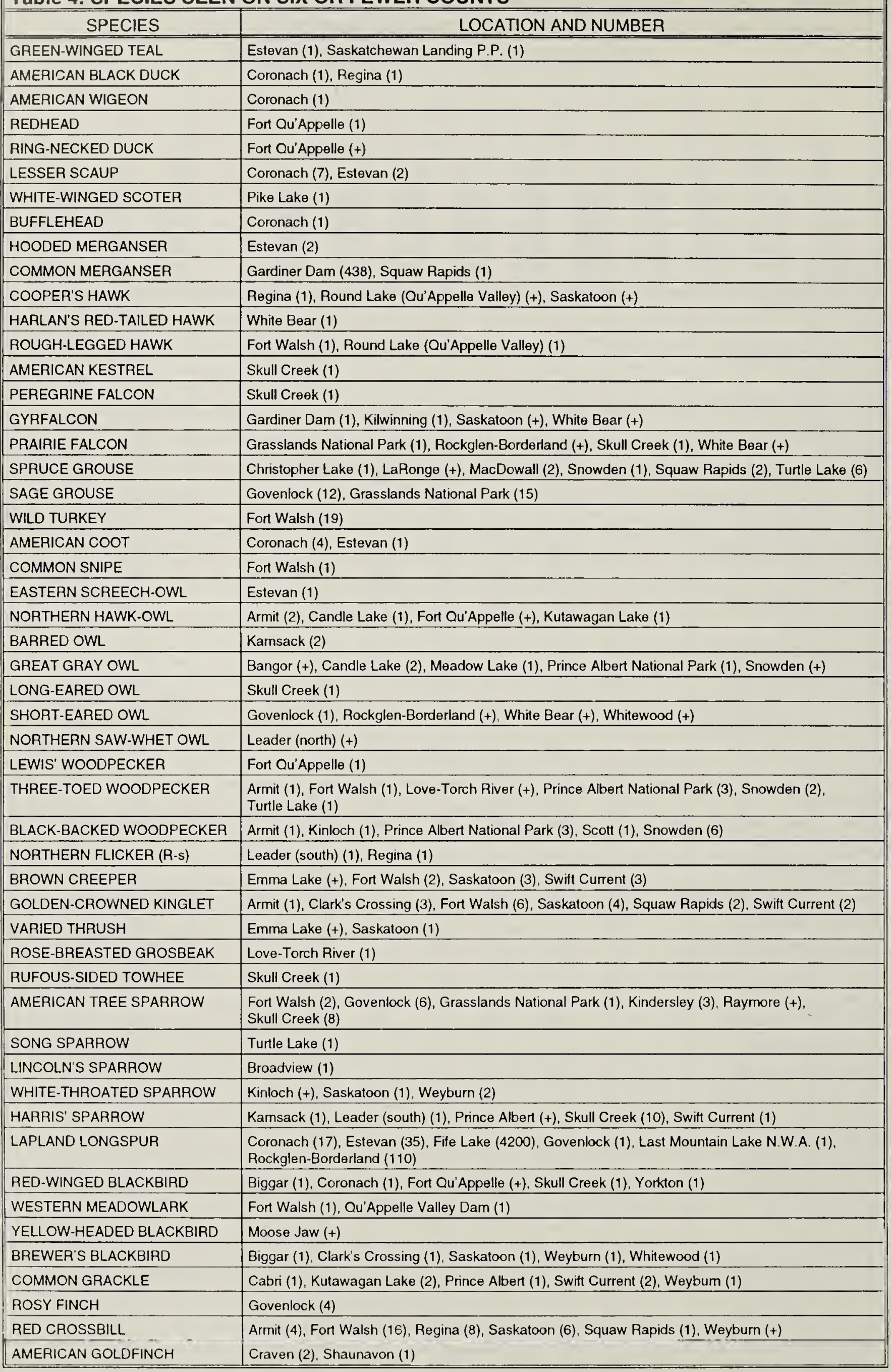




\begin{tabular}{|c|c|c|c|c|c|}
\hline \multicolumn{6}{|c|}{$\begin{array}{l}\text { Table 5: COMPARISON OF THE } 1995 \text { POPULATIONS TO } 1994 \text { AND AVERAGE } \\
\text { PAST YEARS (1990-1994) BASED ON PARTY HOURS PER INDIVIDUAL BIRD SEEN }\end{array}$} \\
\hline SPECIES & 95 vs 94 & $\begin{array}{c}95 \mathrm{vs} \\
5 \mathrm{YR} \\
\end{array}$ & SPECIES & 95 vs 94 & $\begin{array}{c}95 \text { vs } \\
5 Y R \\
\end{array}$ \\
\hline CANADA GOOSE & -77.3 & -51.6 & COMMON RAVEN & -29.6 & -12.7 \\
\hline MALLARD & -63.1 & -10.6 & BLACK-CAPPED CHICKADEE & 14.2 & 23.7 \\
\hline COMMON GOLDENEYE & -54.9 & -26.3 & BOREAL CHICKADEE & -13.3 & 10.2 \\
\hline BALD EAGLE & -81.8 & -54.5 & RED-BREASTED NUTHATCH & 727.5 & 291.0 \\
\hline NORTHERN GOSHAWK & 27.8 & -37.4 & WHITE-BREASTED NUTHATCH & 35.9 & 43.2 \\
\hline GOLDEN EAGLE & -58.6 & -41.8 & BROWN CREEPER & -21.4 & 9.3 \\
\hline MERLIN & -48.6 & -1.0 & GOLDEN-CROWNED KINGLET & -65.3 & -39.2 \\
\hline PRAIRIE FALCON & -73.8 & -79.1 & AMERICAN ROBIN & 13.7 & 20.2 \\
\hline GRAY PARTRIDGE & 173.0 & 57.1 & BOHEMIAN WAXWING & -65.0 & -23.4 \\
\hline RING-NECKED PHEASANT & 41.4 & -17.7 & CEDAR WAXWIN & -85.6 & -61.8 \\
\hline SPRUCE GROUSE & -32.1 & 19.7 & NORTHERN SHRIKE & -36.9 & -30.8 \\
\hline RUFFED GROUSE & -17.3 & 25.1 & EUROPEAN STARLING & -10.2 & 102.5 \\
\hline SHARP-TAILED GROUSE & 79.4 & 19.8 & AMERICAN TREE SPARROW & -31.3 & -41.4 \\
\hline ROCK DOVE & -30.1 & -6.1 & DARK-EYED JUNCO & 416.3 & 281.4 \\
\hline GREAT HORNED OWL & -43.7 & -23.6 & LAPLAND LONGSPUR & 8742.3 & 134.9 \\
\hline SNOWY OWL & -55.6 & -32.9 & SNOW BUNTING & .56 .0 & -32.2 \\
\hline SHORT-EARED OWL & -96.6 & .94 .8 & RED-WINGED BLACKBIRD & .77 .4 & .76 .7 \\
\hline DOWNY WOODDPECKER & 23.6 & 9.8 & RUSTY BLACKBIRD & 85.4 & 68.8 \\
\hline HAIRY WOODPECKER & 16.3 & 21.0 & PINE GROSBEAK & 46.6 & 95.1 \\
\hline THREE-TOED WOODPECKER & -21.4 & 11.7 & RED CROSSBILL & 358.6 & 13.6 \\
\hline NORTHERN FLICKER & -54.3 & 8.8 & WHITE-WINGED CROSSBILL & 0.0 & 536.3 \\
\hline PILEATED WOODPECKER & -56.5 & -40.2 & COMMON REDPOLL & 497.4 & 118.6 \\
\hline HORNED LARK & 286.3 & -13.6 & HOARY REDPOLL & 0.0 & 13.1 \\
\hline GRAY JAY & -18.9 & 7.4 & PINE SISKIN & -100.0 & -100.0 \\
\hline BLUE JAY & 15.7 & 25.9 & EVENING GROSBEAK & 24.2 & 11.3 \\
\hline BLACK-BILLED MAGPIE & -6.2 & 8.6 & HOUSE SPARROW & -26.1 & -16.4 \\
\hline
\end{tabular}

\begin{tabular}{|c|c|c|c|c|}
\hline LOCATION & $\begin{array}{c}1995 \\
\text { COUNT } \\
\end{array}$ & SPECIES & $\begin{array}{c}\text { PREVIOUS } \\
\text { HIGH } \\
\end{array}$ & LOCATION AND YEAR \\
\hline Gardiner Dam & 438 & Commom Merganser & 274 & Gardiner Dam '90 \\
\hline Regina & 1 & Cooper's Hawk & 1 & $\mathrm{~N}$ to Battleford \\
\hline White Bear & 1 & Harlan's Red-tailed Hawk & NEW & \\
\hline Skull Creek & 1 & Peregrine Falcon & 1 & N to Squaw Rapids-Carrot River \\
\hline Estevan & 1 & Eastern Screech-Owl & 1 & Yorkton ' 85 \\
\hline Kamsack & 2 & Barred Owl & 2 & Squaw Rapids ' 88 \\
\hline Fort Qu'Appelle & 1 & Lewis' Woodpecker & NEW & \\
\hline Snowden & 6 & Black-backed Woodpecker & 6 & Squaw Rapids ' 85 \\
\hline Saskatoon & 25 & Northern Flicker & 22 & \begin{tabular}{|l|} 
Saskatoon '94 \\
\end{tabular} \\
\hline Saskatoon & 111 & Blue Jay & 86 & Saskatoon '91 \\
\hline Saskatoon & 670 & Black-billod Magpie & 587 & Saskatoon '90 \\
\hline Saskatoon & 461 & Black-capped Chickadeo & 390 & Saskatoon '91 \\
\hline Saskatoon & 207 & Red-breasted Nuthatch & 88 & Fort Walsh '82 \\
\hline Snowden & 23 & White-breasted Nuthatch & 15 & Round Lake '84, Kamsack '92 \\
\hline [CP: Emma Lake], Saskatoon & 1 & Varied Thrush & 1 & N to Saskatoon \\
\hline Love-Torch River & 1 & Rose-breasted Grosbeak & NEW & \\
\hline Skull Creek & 1 & Rufous-sided Towhee & 1 & N to Leader \\
\hline Broadview & 1 & Lincoln's Sparrow & [1] & [CP: Whitewood '92, Weyburn' 93 ] \\
\hline Grasslands N. Park (NW) & 250 & Dark-eyed Junco & 102 & Swift Current '93 \\
\hline Moose Jaw & 1 & Yellow-headed Blackbird & NEW & \\
\hline Moose Jaw & 44 & House Finch & 22 & Weyburn' 93 \\
\hline Saskatoon & 447 & White-winged Crossbill & 230 & Emma Lake '75 \\
\hline Prince Albert & 214 & Pine Siskin & 70 & Saskatoon '81 \\
\hline Craven & 2 & American Goldfinch & 1 & N to Prince Albert \\
\hline
\end{tabular}




\begin{tabular}{|l|l|}
\hline \multicolumn{2}{|c|}{ SPECIES } \\
\hline HAWK species & Lu'Appelle Valley Dam (1) \\
\hline LARGE FALCON species & Skull Creek (1) \\
\hline REDPOLL species & $\begin{array}{l}\text { Armit (29), Craven (30), Fort Walsh (35), Grasslands National Park (30), } \\
\text { Last Mountain Lake N.W.A. (31), Raymore (250), Regina (13), Squaw Rapids (59) }\end{array}$ \\
\hline BIRD species & Grasslands National Park (NW) (22), Nipawin (9) \\
\hline
\end{tabular}

\section{Count}

The following count was received too late for inclusion in the 1994 compilation.

LOVE-TORCH RIVER. Weather: Temp. -25 to $-20^{\circ} \mathrm{C}$, Wind 0 to $10 \mathrm{kmph}$, Snow depth $50-60 \mathrm{~cm}$, Skies clear. Effort: $35 \mathrm{~km}$ by automobile in 2 hours and 10 hours at feeders. Habitat coverage: $26-50 \%$ mixedwood forest, $<5 \%$ cultivated fields, 5-25\% farmyards, 5-25\% urban. Bird Species: Northern Goshawk +, Spruce Grouse 2, Ruffed Grouse 5, Rock Dove 37, Downy Woodpecker 7, Hairy Woodpecker 12, Three-toed Woodpecker +, Blackbacked Woodpecker +, Pileated Woodpecker 1, Gray Jay 11, Blue Jay 15, Black-billed Magpie 16, Common Raven 16, Black-capped Chickadee 61, White-breasted Nuthatch 7, Dark-eyed Junco +, Snow Bunting 116, Redwinged Blackbird 1, Pine Grosbeak 36, Evening Grosbeak 160, House Sparrow 13. Mammal species: Red Squirrel 3, Red Fox 2, White-tailed Deer 14. Observers: Bert Dalziel (compiler), Joan Dalziel, Kari Dalziel, Sara Dalziel, Betty Donovan, Bruce Donovan, Sean Donovan, George Lidster, Jean Lidster, Bill Matthews, Lynn Matthews. (11)

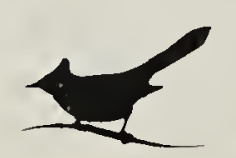

Biological diversity - "biodiversity" in the new parlance - is the key to the maintenance of the world as we know it. Life in a local site struck down by a passing storm springs back quickly because enough diversity still exists. Opportunistic species evolved for just such an occasion rush in to fill the spaces. They entrain the succession that circles back to something resembling the original state of the environment. E.O. Wilson, 1992. The diversity of life. W.W. Norton and Company, New York. 424 pp. 